\title{
OPTIMAL CONTROL OF A STOCHASTIC HEAT EQUATION WITH BOUNDARY-NOISE AND BOUNDARY-CONTROL
}

\author{
Arnaud Debussche ${ }^{1}$, Marco Fuhrman $^{2}$ and Gianmario Tessitore $^{3}$
}

\begin{abstract}
We are concerned with the optimal control of a nonlinear stochastic heat equation on a bounded real interval with Neumann boundary conditions. The specificity here is that both the control and the noise act on the boundary. We start by reformulating the state equation as an infinite dimensional stochastic evolution equation. The first main result of the paper is the proof of existence and uniqueness of a mild solution for the corresponding Hamilton-Jacobi-Bellman (HJB) equation. The $C^{1}$ regularity of such a solution is then used to construct the optimal feedback for the control problem. In order to overcome the difficulties arising from the degeneracy of the second order operator and from the presence of unbounded terms we study the HJB equation by introducing a suitable forward-backward system of stochastic differential equations as in the appraoch proposed in [14,27] for finite dimensional and infinite dimensional semilinear parabolic equations respectively.
\end{abstract}

Mathematics Subject Classification. 60H30, 49L20, 93E20, 35K20.

Received October 26, 2004. Revised December 7, 2005.

\section{INTRODUCTION}

We are here concerned with an optimal control problem for a state equation of parabolic type on a bounded real interval, which for convenience we take equal to $[0, \pi]$. We stress the fact that we consider Neumann boundary conditions in which the derivative of the unknown is equal to the sum of the control and of a white

\footnotetext{
Keywords and phrases. Boundary noise, optimal boundary control, HJB equation, backward stochastic differential equations.

1 ENS Cachan, Antenne de Bretagne, Campus de Ker Lann, 35170, Bruz Cedex, France;

Arnaud.Debussche@bretagne.ens-cachan.fr

2 Dipartimento di Matematica, Politecnico di Milano, piazza Leonardo da Vinci 32, 20133 Milano, Italy; marco.fuhrman@polimi.it

Partially supported by the European Community's Human Potential Programms under contracts HPRN-CT-2002-00279, QP-Applications and HPRN-CT-2002-00281, Evolution Equations.

3 Dipartimento di Matematica e Applicazioni, Università di Milano-Bicocca, via R. Cozzi 53 - Edificio U5, 20125 Milano, Italy; gianmario.tessitore@unimib.it

Research supported by European Community's Human Potential Programm under contract HPRN-CT-2002-00281, Evolution Equations.

(C) EDP Sciences, SMAI 2007
} 
noise in time, namely:

$$
\left\{\begin{array}{l}
\frac{\partial y}{\partial s}(s, \xi)=\frac{\partial^{2} y}{\partial \xi^{2}}(s, \xi)+f(s, y(s, \xi)), \quad s \in[t, T], \xi \in(0, \pi) \\
y(t, \xi)=x(\xi) \\
\frac{\partial y}{\partial \xi}(s, 0)=u^{1}(s)+\dot{W}_{s}^{1}, \quad \frac{\partial y}{\partial \xi}(s, \pi)=u^{2}(s)+\dot{W}_{s}^{2}
\end{array}\right.
$$

In the above equation $\left\{W_{t}^{i}, t \geq 0\right\}, i=1,2$, are independent standard real Wiener processes; the unknown $y(s, \xi, \omega)$, representing the state of the system, is a real-valued process; the control is modellized by the two predictable real-valued processes $u^{i}(s, \xi, \omega), i=1,2$ acting, respectively, at 0 and $\pi ; x$ is in $L^{2}(0, \pi)$.

Most of existing articles on stochastic control deal with distributed parameter controls (see for instance [15] and the references therein). This is not a realistic situation since in practice such controls are not easy to implement. We think that boundary controls are much more natural. Such control problems have been widely studied in the deterministic literature [22] and have been addressed in the stochastic case as well (see $[10,19,21,23])$. However, in these works, the equation always contains noise as a forcing term as well. We stress the fact that in this latter situation the treatment of the Hamilton-Jacobi equation is easier since the presence of enough noise guaranties that the linear operator in the Hamilton-Jacobi equation is strongly elliptic. Here, we consider the limit and more difficult situation of a noise acting only at the boundary. We could consider as well a supplementary noise in the equation. Our problem corresponds to a realistic situation where the controls itself is perturbed by a noise.

The present article is a first step of our program. Indeed, we consider a simple nonlinear heat equation and a Neumann boundary conditions. Such model arise naturally to describe chemical reactions for instance.

Our techniques seem to applicable to an equation like Burgers equation but several technical difficulties appear. An example of physically realistic situation which we have in mind is the control of a fluid in the context of thermo-hydraulics. The Boussinesq equations can be used to model the evolution of the velocity and temperature of the fluid. It is very natural to control the temperature or the flux of temperature of the boundary. If the control is perturbed by a noise, we probably can use our method. These problems will be the object of future work. The case of Dirichlet boundary conditions is more complicated since the solutions are much less regular in space. It is possible that our method can be extended to this case using the techniques of $[4,29]$. Roughly speaking, the real structural restriction - beside technical complications - is that the "image of the noise operator" is larger than the image of the control.

Several works have been devoted to stochastic partial differential equations with noise on the boundary (for early results see, for instance, $[2,7,21,28]$ ). An important contribution is given in [29] where the behavior, close to the boundary, of the solutions to a multidimensional parabolic equation with Neumann boundary conditions is studied. In the same spirit are the results in $[3,4]$ where the Dirichlet boundary conditions are treated.

A more abstract approach, based on semigroup theory, is used in [8] (see also [9] and [23]). We notice that in [8] it is also shown that, if we replace Neumann by Dirichlet boundary conditions, the solutions of (1.1) are not square integrable in space in general. The solutions are singular at the boundary, the singularity is described in [3] and [29]. The reason is that the smoothing properties of the heat equation are not strong enough to regularize a rough term such as a white noise.

We finally notice that [4] and [23] are essentially devoted to the long time behavior of the solutions of stochastic equations with noise on the boundary.

Here we follow [8] and we reformulate equation (1.1) as a stochastic evolution equation in the infinite dimensional Hilbert space $H=L^{2}(0, \pi)$ in Section 2.2. Namely we rewrite it as:

$$
\left\{\begin{array}{l}
\frac{\mathrm{d}}{\mathrm{d} s} X_{s}=A X_{s}+F\left(s, X_{s}\right)+\sum_{i=1}^{2}(\lambda-A) b^{i} \dot{W}_{s}^{i}+\sum_{i=1}^{2}(\lambda-A) b^{i} u^{i}(s) \mathrm{d} s, \quad s \in[t, T], \\
X_{t}=x
\end{array}\right.
$$


where $A$ stands for the Laplace operator with homogeneous Neumann boundary conditions, $b^{1}, b^{2}$ are suitable functions in $L^{2}(0, \pi), F$ is the evaluation operator corresponding to $f$ and $\lambda$ is an arbitrary positive number.

The optimal control problem we wish to treat in this paper consists in minimizing the following finite horizon cost

$$
J\left(t, x, u^{1}(\cdot), u^{2}(\cdot)\right)=\mathbb{E} \int_{t}^{T} \int_{0}^{\pi} \ell\left(s, \xi, y(s, \xi), u^{1}(s), u^{2}(s)\right) \mathrm{d} \xi \mathrm{d} s+\mathbb{E} \int_{0}^{\pi} \phi(\xi, y(T, \xi)) \mathrm{d} \xi .
$$

Our purpose is not only to prove existence of optimal controls but mainly to characterize them by an optimal feedback law. In other words we wish to perform the standard program of synthesis of the optimal control that consists in the following steps: first we solve (in a suitable sense) the Hamilton-Jacobi-Bellman equation; then we prove that such a solution is the value function of the control problem and allows to construct the optimal feedback law.

As far as existence of optimal controls is concerned this was obtained in [10] for an equation similar to ours (but with an additional non degenerate diffusion noise term) and for an ergodic cost functional (that is a functional that depends on the asymptotic behavior of the state). We notice that the authors exploit there the smoothing properties of the Kolmogorov equation associated with the state equation that, in their case, is non-degenerate.

Here the main object of investigation will be the following (formally written) Hamilton-Jacobi-Bellman equation:

$$
\left\{\begin{array}{l}
\frac{\partial v(t, x)}{\partial t}+\mathcal{L}_{t}[v(t, \cdot)](x)=\Psi\left(t, x, \nabla v(t, x)(\lambda-A) b^{1}, \nabla v(t, x)(\lambda-A) b^{2}\right), \quad t \in[0, T], x \in H, \\
v(T, x)=\Phi(x)
\end{array}\right.
$$

where $\mathcal{L}_{t}$ is the infinitesimal generator of the Markov semigroup corresponding to the process $y$ :

$$
\mathcal{L}_{t}[\phi](x)=\frac{1}{2}\left\langle\nabla^{2} \phi(x)(\lambda-A) b^{1},(\lambda-A) b^{1}\right\rangle+\frac{1}{2}\left\langle\nabla^{2} \phi(x)(\lambda-A) b^{2},(\lambda-A) b^{2}\right\rangle+\langle A x+F(t, x), \nabla \phi(x)\rangle .
$$

In (1.4), $\Psi$ is the hamiltonian function of the problem, defined in terms of $\ell$ (see Sect. 2.3), and $\Phi$ is the evaluation map corresponding to $\phi$.

We notice that two different kinds of difficulties add up in above equation. Firstly $\mathcal{L}_{t}$ is highly degenerate, indeed $\nabla^{2} \phi(x)$ appears only multiplied by $(\lambda-A) b^{i}, i=1,2$. Therefore, the Kolmogorov equation associated with the state equation has very poor smoothing properties. Moreover unbounded terms are involved both in the operator $\mathcal{L}_{t}$ and in the nonlinearity $\Psi$.

We formulate the equation (1.4) in a mild sense, see for instance [17] and [18]. Namely we set $\left\{P_{t, s}[\cdot]: 0 \leq\right.$ $s \leq t\}$, to be the Markov semigroup corresponding to equation (1.1) and we seek a function $v:[0, T] \times H \rightarrow \mathbb{R}$ verifying the following variation of constants formula:

$$
v(t, x)=P_{t, T}[\Phi](x)-\int_{t}^{T} P_{t, s}\left[\Psi\left(s, \cdot \nabla v(s, \cdot)(\lambda-A) b^{1}, \nabla v(s, \cdot)(\lambda-A) b^{2}\right)\right](x) \mathrm{d} s, \quad t \in[0, T], x \in H .
$$

We notice that, when the state equation is linear, it is known that the semigroup $\left\{P_{s, t}[\cdot]: 0 \leq s \leq t\right\}$ is strongly Feller (see [8]). It maps bounded and measurable functions into differentiable functions. Let $C_{t, s}$ be a constant verifying, for all bounded and measurable functions $v, \sup _{x \in H}\left|\nabla P_{t, s} v(x)\right| \leq C_{t, s} \sup _{x \in H}|v(x)|$. If we try to estimate the constant $C_{t, s}$ using the minimal energy null controllability characterization (see for instance [9], Chap. 7.2) and Carleman estimates (see for instance [1] or [16]), the best bound we can obtain explodes exponentially as $t$ approaches to $s$. Thus, even in the linear case, it seems impossible to solve equation (1.5) by a fixed point argument in a set of differentiable functions as it is done, for instance, in [17] or [18].

We also mention here that, as it is well known, when $H$ is finite dimensional Hamilton-Jacobi-Bellman equations can be successfully treated using the notion of viscosity solution. The point is that, in the infinite dimensional case, very few uniqueness results are available for viscosity solutions and all of them, obtained by 
analytic techniques, impose strong assumptions on operator $G$ and on the nonlinearity $\Psi$, see, for instance, [19] or [30] and references within.

Another approach has been used more recently to solve this kind of equation. It is based on the use of a system of Forward-Backward stochastic differential equations and generalizes the finite dimensional method developed in the fundamental papers $[11,26,27]$. This approach requires a structural property on the equation. Roughly speaking, it requires that the control acts only where the noise acts. It is obviously satisfied in our case.

The backward stochastic differential equation is in our case

$$
\left\{\begin{array}{l}
\mathrm{d} Y_{s}=\Psi\left(s, X(s, t, x), Z_{s}^{1}, Z_{s}^{2}\right) \mathrm{d} s+Z_{s}^{1} \mathrm{~d} W_{s}^{1}+Z_{s}^{2} \mathrm{~d} W_{s}^{2}, \quad s \in[t, T] \\
Y_{T}=\Phi(X(T, t, x))
\end{array}\right.
$$

where $X(s, t, x)$ stands for the solution of equation (1.2) starting at time $t$ from $x \in H$. We set $v(t, x)=Y_{t}$. It is classical that, formally, $v$ is a solution of equation (1.4). Our purpose is to prove this rigorously and, more precisely, to show that $v$ is the unique mild solution of equation (1.4).

The difficulty is that, contrary to the case treated in [14] and [15], it is not enough to prove that $v$ is once (Gâteaux) differentiable to give sense to equation (1.4). Indeed the occurrence of the term $\nabla v(s, \cdot)(\lambda-A) b^{i}$, $i=1,2$, together with the fact that $b^{i}$ do not belong to $D(A)$ but only to $D(-A)^{\alpha}$, for $\alpha<3 / 4$, forces us to prove that the map $(t, s, h) \rightarrow \nabla v(t, x)(\lambda-A)^{\beta} h$ extends, for $\beta<1 / 2$, to a continuous map on $[0, T] \times H \times H$. To do that we start proving that this extra regularity holds, in a suitable sense, for the forward equation (1.2) and then that it is conserved if we differentiate (in Gâteaux sense) the backward equation with respect to the process $X$.

On the other side, showing first that $X(\cdot, t, x)$ is regular in Malliavin sense, we can prove that if the map $(t, s, h) \rightarrow \nabla v(t, x)(\lambda-A)^{\beta} h$ extends to a continuous function on $[0, T] \times H \times H$ then the processes $s \rightarrow$ $v(s, X(s, t, x))$ and $W^{i}, i=1,2$ admit joint quadratic variation in any interval $[t, \tau]$ and this is given by $\int_{t}^{\tau} \nabla v(s, X(s))(\lambda-A) b^{i} \mathrm{~d} s, i=1,2$. As a consequence we are able to identify the right class of functions in which our candidate solution is contained and for which (1.4) is meaningful, see Definition 5.1. Then we proceed as in [15] exploiting the probabilistic definition of $v$ and the characterization of $\int_{t}^{\tau} Z^{i}(s) \mathrm{d} s$ as joint quadratic variation between $Y$ and $W^{i}$ in $[s, \tau]$ to prove that $v$ is the unique mild solution of (1.4), see Theorem 5.1.

We can then come back to the control problem and using the probabilistic representation of the unique mild solution to equation (1.4) we easily show existence of an optimal feedback law, see Theorem 6.1. We finally notice that the lack of regularity of the feedback does not allow to prove existence of a strong solution to the closed loop equation. On the contrary, if we formulate the control problem in a suitable weak sense we can ensure existence of an optimal control, satisfying the optimal feedback law, see Theorem 6.4.

We think that it is important for the applications to treat optimal control with control and noise at the boundary. In the present article, we treat a problem where all the coefficients are Lipschitz functions. This is clearly a restriction. In forthcoming work, we shall treat more realistic situations such as the Burgers equation controlled by the boundary.

The paper is structured as follows: in Section 2 we transpose the problem in the infinite dimensional framework; in Section 3 we study the (forward) state equation and in Section 4 the backward equation associated to the problem; in Section 5 we prove existence and uniqueness of the Hamilton-Jacobi-Bellman partial differential equation and in Section 6 we show how the previous results can be applied to perform the synthesis of the optimal control when the control problem is formulated in a strong sense (Sect. 6.1) and when a weak formulation is chosen (Sect. 6.2). 


\section{Statement AND Reformulation of the Problem}

\subsection{General assumptions}

In equation (1.1) $\left\{W_{t}^{i}, t \geq 0\right\}, i=1,2$, are independent standard real Wiener processes on a probability space $(\Omega, \mathcal{F}, \mathbb{P})$, with respect to a filtration $\left\{\mathcal{F}_{t}, t \geq 0\right\}$ satisfying the usual conditions. Also, $u=\left(u^{1}, u^{2}\right)$ : $\Omega \times[0, T] \rightarrow \mathbb{R}^{2}$ is the control process; it is called admissible if it is $\left(\mathcal{F}_{t}\right)$-predictable and takes values in a set $\mathcal{U} \subset \mathbb{R}^{2}$, called the set of control actions.

We assume the following.

\section{Hypothesis 2.1.}

(1) The function $f:[0, T] \times \mathbb{R} \rightarrow \mathbb{R}$ is measurable, for every $t \in[0, T]$ the function $f(t, \cdot): \mathbb{R} \rightarrow \mathbb{R}$ is continuously differentiable and there exists a constant $C_{f}$ such that

$$
|f(t, 0)|+\left|\frac{\partial f}{\partial r}(t, r)\right| \leq C_{f}, \quad t \in[0, T], r \in \mathbb{R} .
$$

(2) The initial condition $x(\cdot)$ belongs to $L^{2}(0, \pi)$.

(3) The set of control actions $\mathcal{U}$ is a bounded closed subset of $\mathbb{R}^{2}$.

Concerning the functions $\ell$ and $\phi$ appearing in the cost functional we make the following general assumptions.

Hypothesis 2.2. $\ell:[0, T] \times[0, \pi] \times \mathbb{R} \times \mathcal{U} \rightarrow \mathbb{R}$ and $\phi:[0, \pi] \times \mathbb{R} \rightarrow \mathbb{R}$ are measurable and the following holds:

(1) There exists a constant $C_{\phi}$ such that

$$
\left|\phi\left(\xi, y_{1}\right)-\phi\left(\xi, y_{2}\right)\right| \leq C_{\phi}\left(1+\left|y_{1}\right|+\left|y_{2}\right|\right)\left|y_{1}-y_{2}\right|, \quad \xi \in[0, \pi], y_{1}, y_{2} \in \mathbb{R} .
$$

Moreover $\int_{0}^{\pi}|\phi(\xi, 0)| \mathrm{d} \xi<\infty$.

(2) For every $t \in[0, T]$ and $\xi \in[0, \pi], \ell(t, \xi, \cdot, \cdot, \cdot): \mathbb{R}^{3} \rightarrow \mathbb{R}$ is continuous. Moreover there exists a constant $C_{\ell}$ such that for every $t \in[0, T], \xi \in[0, \pi], y_{1}, y_{2} \in \mathbb{R},\left(u^{1}, u^{2}\right) \in \mathcal{U}$,

$$
\left|\ell\left(t, \xi, y_{1}, u^{1}, u^{2}\right)-\ell\left(t, \xi, y_{2}, u^{1}, u^{2}\right)\right| \leq C_{\ell}\left(1+\left|y_{1}\right|+\left|y_{2}\right|\right)\left|y_{1}-y_{2}\right|,
$$

and for every $t \in[0, T]$

$$
\int_{0}^{\pi} \sup _{\left(u^{1}, u^{2}\right) \in \mathcal{U}}\left|\ell\left(t, \xi, 0, u^{1}, u^{2}\right)\right| \mathrm{d} \xi \leq C_{\ell} .
$$

Further assumptions will be made on the cost functional after its reformulation: see Hypothesis 2.6 below.

\subsection{Reformulation of the state equation. Existence and uniqueness}

It is our purpose to write the state equation as an evolution equation in the space $H=L^{2}(0, \pi)$. This is done for instance in [9] and we follow that approach. We recall that the semigroup approach to boundary value problems goes back to the pioneering works by Fattorini [12] and Balakrishnan [6] (see also [22]). We briefly sketch the argument for the reader's convenience and in order to fix some notation.

The state will be denoted by $X_{s}^{u}=y(s, \cdot)$. Thus $\left\{X_{s}^{u}, s \in[t, T]\right\}$ is a process in $H$ and the initial condition is assumed to belong to $H$.

We define the operator $A$ in $H$ setting

$$
D(A)=\left\{y \in H^{2}(0, \pi): \frac{\partial y}{\partial \xi}(0)=\frac{\partial y}{\partial \xi}(\pi)=0\right\}, \quad A y=\frac{\partial^{2} y}{\partial \xi^{2}} \text { for } y \in D(A) .
$$


The operator $A$ is self-adjoint with eigenvalues $\lambda_{n}=-n^{2}, n=0,1, \ldots$ The domains of the fractional powers of $-A$ are related to the Sobolev spaces of fractional order as follows:

$$
\begin{array}{lll}
D(-A)^{\alpha}=\left\{y \in H^{2 \alpha}(0, \pi): \frac{\partial y}{\partial \xi}(0)=\frac{\partial y}{\partial \xi}(\pi)=0\right\}, & \text { for } 3 / 4<\alpha<1, \\
D(-A)^{\alpha}=H^{2 \alpha}(0, \pi), & & \text { for } 0<\alpha<3 / 4 .
\end{array}
$$

We define $F:[0, T] \times H \rightarrow H$ setting $F(t, x)=f(t, x(\cdot))$ for $x \in H$. Clearly, $F$ is well defined under our assumptions.

We fix $\lambda>0$ and define

$$
b^{1}(\xi)=-\frac{\cosh (\sqrt{\lambda}(\pi-\xi))}{\sqrt{\lambda} \sinh (\sqrt{\lambda} \pi)}, \quad b^{2}(\xi)=\frac{\cosh (\sqrt{\lambda} \xi)}{\sqrt{\lambda} \sinh (\sqrt{\lambda} \pi)}, \quad \xi \in[0, \pi],
$$

and note that they solve the Neumann problems

$$
\left\{\begin{array}{l}
\frac{\partial^{2} b^{i}}{\partial \xi^{2}}(\xi)=\lambda b^{i}(\xi), \quad \xi \in(0, \pi), i=1,2, \\
\frac{\partial b^{1}}{\partial \xi}(0)=1, \quad \frac{\partial b^{1}}{\partial \xi}(\pi)=0 \\
\frac{\partial b^{2}}{\partial \xi}(0)=0, \quad \frac{\partial b^{2}}{\partial \xi}(\pi)=1 .
\end{array}\right.
$$

Thus $b^{i} \in D(-A)^{\alpha}$ for $0<\alpha<3 / 4, b^{i} \notin D(-A)^{\alpha}$ for $3 / 4<\alpha<1$.

Equation (1.1) can now be reformulated as

$$
\left\{\begin{array}{l}
\frac{\mathrm{d}}{\mathrm{d} s} X_{s}^{u}=A X_{s}^{u}+F\left(s, X_{s}^{u}\right)+(\lambda-A) b \dot{W}_{s}+(\lambda-A) b u(s), \quad s \in[t, T], \\
X_{0}^{u}=x
\end{array}\right.
$$

where we have defined

$$
W=\left(\begin{array}{l}
W^{1} \\
W^{2}
\end{array}\right), \quad u(t)=\left(\begin{array}{c}
u^{1}(t) \\
u^{2}(t)
\end{array}\right), \quad b=\left(b^{1}(\cdot), \quad b^{2}(\cdot)\right) .
$$

The proof of the equivalence between equation (1.1) and equation (2.1) can be founded, for instance, in [9] and [7]. We briefly report here the formal argument (for simplicity take $f=0$ ).

Let $v(s, \xi)=b^{1}(\xi)\left(u^{1}(s)+\dot{W}_{s}^{1}\right)-b^{2}(\xi)\left(u^{2}(s)+\dot{W}_{s}^{2}\right)$ and let $\ell(s, \xi)=y(s, \xi)-v(s, \xi)$. We notice that $\frac{\partial \ell_{s}}{\partial \xi}(0)=\frac{\partial \ell_{s}}{\partial \xi}(\pi)=0$, consequently $\ell_{s} \in D(A)$ (we assume that $y_{s} \in H^{2}(0, \pi)$ ). Differentiating $\ell$ with respect to time we get

Therefore

$$
\frac{\partial \ell}{\partial s}(s, \xi)=\frac{\partial^{2} \ell}{\partial \xi^{2}}(s, \xi)+\lambda v(s, \xi)-\frac{\mathrm{d}}{\mathrm{d} s} v(\xi, s)=\left(A \ell_{s}\right)(\xi)+\lambda v(s, \xi)-\frac{\mathrm{d}}{\mathrm{d} s} v(\xi, s)
$$

$$
y_{t}=v_{t}+\ell_{t}=v_{t}+\mathrm{e}^{t A}\left(x-v_{0}\right)+\lambda \int_{0}^{t} \mathrm{e}^{(t-s) A} v_{s} \mathrm{~d} s-\int_{0}^{t} \mathrm{e}^{(t-s) A} \frac{\mathrm{d}}{\mathrm{d} s} v_{s} \mathrm{~d} s .
$$

Now, if we use integration by parts formula to rewrite the last term, we obtain the desired relation, namely:

$$
y_{t}=\mathrm{e}^{t A} x+\lambda \int_{0}^{t} \mathrm{e}^{(t-s) A} v_{s} \mathrm{~d} s-\int_{0}^{t} A \mathrm{e}^{(t-s) A} v_{s} \mathrm{~d} s .
$$

At this moment it is convenient to list the relevant properties of the objects introduced so far in this section. Therefore we formulate the following proposition. 
Proposition 2.3. We fix $\lambda>0, \beta \in(1 / 2,3 / 4)$. Under Hypothesis 2.1 the following properties hold:

(1) $H$ is a real separable Hilbert space;

(2) $A$ is a self-adjoint, non positive operator in $H$;

(3) $b^{1}, b^{2}$ are elements of $D(\lambda-A)^{\beta}$; we set $b=\left(b^{1}, b^{2}\right)$;

(4) $F:[0, T] \times H \rightarrow H$ is a measurable function and there exists a constant $C_{F}$ such that

$$
|F(t, 0)| \leq C_{F}, \quad\left|F\left(t, x_{1}\right)-F\left(t, x_{2}\right)\right| \leq C_{F}\left|x_{1}-x_{2}\right|, \quad t \in[0, T], x, x_{1}, x_{2} \in H .
$$

Moreover, for every $t \in[0, T], F(t, \cdot)$ has a Gâteaux derivative $\nabla F(t, x)$ at every point $x \in H$. Finally, the function $(x, h) \rightarrow \nabla F(t, x) h$ is continuous as a map $H \times H \rightarrow \mathbb{R}$.

Note that we consider $\nabla F(t, x)$ as an element of $H^{*}$ and we denote its action on $h \in H$ by $\nabla F(t, x) h$. It follows from the assumptions that $|\nabla F(t, x)| \leq C_{F}$ for $t \in[0, T], x \in H$.

We note that $W$ and $u$ remain as in the previous section: $W$ is a standard Wiener processes in $\mathbb{R}^{2}$ on a probability space $(\Omega, \mathcal{F}, \mathbb{P})$, endowed with a filtration $\left\{\mathcal{F}_{t}, t \geq 0\right\}$ satisfying the usual conditions, and the control process $u$ is an $\left(\mathcal{F}_{t}\right)$-predictable process with values in a bounded closed set $\mathcal{U} \subset \mathbb{R}^{2}$.

The equation (2.1) is formal. The precise meaning of the state equation is as follows: we say that a continuous, $H$-valued, predictable process $X^{u}$ is a solution of the state equation if, $\mathbb{P}$-a.s.,

$$
\begin{aligned}
X_{s}^{u}= & \mathrm{e}^{(s-t) A} x+\int_{t}^{s} \mathrm{e}^{(s-r) A} F\left(r, X_{r}^{u}\right) \mathrm{d} r+\int_{t}^{s}(\lambda-A)^{1-\beta} \mathrm{e}^{(s-r) A}(\lambda-A)^{\beta} b \mathrm{~d} W_{r} \\
& +\int_{t}^{s}(\lambda-A)^{1-\beta} \mathrm{e}^{(s-r) A}(\lambda-A)^{\beta} b u(r) \mathrm{d} r, \quad s \in[t, T] .
\end{aligned}
$$

The stochastic integral in $(2.2)$ is

$$
\int_{t}^{s}(\lambda-A)^{1-\beta} \mathrm{e}^{(s-r) A}(\lambda-A)^{\beta} b^{1} \mathrm{~d} W_{r}^{1}+\int_{t}^{s}(\lambda-A)^{1-\beta} \mathrm{e}^{(s-r) A}(\lambda-A)^{\beta} b^{2} \mathrm{~d} W_{r}^{2},
$$

where each term is an Ito stochastic integral of an $H$-valued deterministic integrand with respect to a real Wiener process. It is well defined since $b^{i} \in D(\lambda-A)^{\beta}$ and therefore

$$
\begin{aligned}
\int_{t}^{s}\left|(\lambda-A)^{1-\beta} \mathrm{e}^{(s-r) A}(\lambda-A)^{\beta} b^{i}\right|^{2} \mathrm{~d} r & \leq \int_{t}^{s}\left|(\lambda-A)^{1-\beta} \mathrm{e}^{(s-r) A}\right|^{2}\left|(\lambda-A)^{\beta} b^{i}\right|^{2} \mathrm{~d} r \\
& \leq\left|(\lambda-A)^{\beta} b^{i}\right|^{2} \int_{t}^{s} c(s-r)^{2 \beta-2} \mathrm{~d} r<\infty,
\end{aligned}
$$

since $\beta>1 / 2$.

Existence and uniqueness of the solution of (2.2) is asserted in Proposition (2.4) below. It is also useful to consider the uncontrolled version of equation (2.2) namely:

$$
X_{s}=\mathrm{e}^{(s-t) A} x+\int_{t}^{s} \mathrm{e}^{(s-r) A} F\left(r, X_{r}\right) \mathrm{d} r+\int_{t}^{s}(\lambda-A)^{1-\beta} \mathrm{e}^{(s-r) A}(\lambda-A)^{\beta} b \mathrm{~d} W_{r}, \quad s \in[t, T] .
$$

We will refer to $(2.3)$ as the forward equation.

In the following, if $K$ is a separable subspace of $H$, by $L_{\mathcal{P}}^{p}(\Omega ; C([0, T] ; K))$ we denote the space of the adapted continuous processes $Y$ in $K$ such that $\mathbb{E} \sup _{s}\left|Y_{s}\right|_{K}^{p}<\infty$.

Proposition 2.4. Under the assumptions in Hypothesis 2.3, for an arbitrary $\mathcal{U}$ valued predictable process $u$, for every $t \in[0, T]$ and $x \in H$ there exists a unique adapted process $\left\{X_{s}^{u}, s \in[0, T]\right\}$ (respectively $\left\{X_{s}, s \in[0, T]\right\}$ ) 
with continuous trajectories, solving (2.2) (respectively (2.3)). Moreover for every $p \in[1, \infty), \alpha \in[0,1 / 4)$, $t \in[0, T]$ there exists a constant $c_{p, \alpha}$ such that

$$
\mathbb{E} \sup _{s \in(t, T]}(s-t)^{p \alpha}|X(s, t, x)|_{D(-A)^{\alpha}}^{p} \leq c_{p, \alpha}\left(1+|x|_{H}\right)^{p}
$$

Proof. Let us define, for $s \in[t, T]$,

$$
\begin{aligned}
& V_{1}(s, t)=\int_{t}^{s}(\lambda-A)^{1-\beta} \mathrm{e}^{(s-r) A}(\lambda-A)^{\beta} b \mathrm{~d} W_{r}, \\
& V_{2}(s, t)=\int_{t}^{s}(\lambda-A)^{1-\beta} \mathrm{e}^{(s-r) A}(\lambda-A)^{\beta} b u(r) \mathrm{d} r .
\end{aligned}
$$

The state equation (2.2) can be written

$$
X_{s}=\mathrm{e}^{(s-t) A} x+\int_{t}^{s} \mathrm{e}^{(s-r) A} F\left(r, X_{r}\right) \mathrm{d} r+V_{1}(s, t)+V_{2}(s, t), \quad s \in[t, T] .
$$

Fix $\alpha \in[0,1 / 4)$. We start by proving that, for all $t \in[0, T]$ and $p \in\left[1,+\infty\left[, V_{1}(\cdot, t) \in L_{\mathcal{P}}^{p}\left(\Omega ; C\left([0, T] ; D(-A)^{\alpha}\right)\right.\right.\right.$. This will be done by the usual factorization procedure (see [9]).

We choose $\beta<3 / 4, \gamma<1 / 2$ and $p$ large enough such that $\alpha+1-\beta+p^{-1}<\gamma<1 / 2$ and define:

$$
y_{\sigma}=\int_{t}^{\sigma}(\sigma-\tau)^{-\gamma} \mathrm{e}^{(\sigma-\tau) A}(\lambda-A)^{\beta} b \mathrm{~d} W_{\tau}
$$

Since $(\lambda-A)^{\beta} b \in L\left(\mathbb{R}^{2}, H\right)$ the Burkholder-Davis-Gundy inequality yields: $\mathbb{E} \int_{t}^{T}\left|y_{\sigma}\right|^{p} \mathrm{~d} \sigma<+\infty$. In particular $y \in L^{p}([t, T] ; H), \mathbb{P}$-a.s. Moreover if we set

$$
\left(R_{\gamma} \ell\right)(s)=\int_{t}^{s}(s-\sigma)^{\gamma-1}(\lambda-A)^{\alpha+1-\beta} \mathrm{e}^{(s-\sigma) A} \ell(\sigma) \mathrm{d} \sigma
$$

then in [9], Proposition A.1.1, it is proved that $R_{\gamma}$ is a bounded linear operator from $L^{p}([t, T] ; H)$ to $C([t, T] ; H)$. Finally by stochastic Fubini-Tonelli Theorem we can rewrite:

$$
\begin{aligned}
\left(R_{\gamma} y\right)(s) & =\int_{t}^{s} \int_{t}^{\sigma}(s-\sigma)^{\gamma-1}(\sigma-\tau)^{\gamma}(\lambda-A)^{\alpha+1} \mathrm{e}^{(s-\tau) A} b \mathrm{~d} W_{\tau} \mathrm{d} \sigma \\
& =\left[\int_{0}^{1}(1-\sigma)^{\gamma-1} \sigma^{-\gamma} \mathrm{d} \sigma\right](\lambda-A)^{\alpha} V_{1}(s, t)
\end{aligned}
$$

and conclude that $V_{1}(\cdot, t) \in L_{\mathcal{P}}^{p}\left(\Omega ; C\left([0, T] ; D(-A)^{\alpha}\right)\right)$.

In a similar (and easier) way it is possible to show that $V_{2}(\cdot, t) \in L_{\mathcal{P}}^{p}\left(\Omega ; C\left([0, T] ; D(-A)^{\alpha}\right)\right)$.

For $a>0$ we denote by $\mathbb{K}_{a, \alpha, t}$ the Banach space of all predictable processes $X: \Omega \times(t, T] \rightarrow D(-A)^{\alpha}$ such that

$$
|X|_{\mathbb{K}_{a, \alpha, t}}^{p}:=\mathbb{E} \sup _{s \in(t, T]} \mathrm{e}^{\text {pas }}(s-t)^{p \alpha}\left|X_{s}\right|_{D(-A)^{\alpha}}^{p}<+\infty
$$

endowed with the above norm. We have just shown that $V_{i}(\cdot, t) \in \mathbb{K}_{a, \alpha, t}, i=1,2$. Moreover, for all $x \in H$,

$$
\sup _{s \in(t, T]}(s-t)^{\alpha}\left|\mathrm{e}^{(s-t) A} x\right|_{D(-A)^{\alpha}} \leq c|x| .
$$


Thus if we define for $X \in \mathbb{K}_{a, \alpha, t}$

$$
\Lambda(X, t)(s)=\int_{t}^{s} \mathrm{e}^{(s-r) A} F\left(r, X_{r}\right) \mathrm{d} r+\mathrm{e}^{(s-t) A} x+V_{1}(s, t)+V_{2}(s, t),
$$

it is immediate to prove that $\Lambda(X, t) \in \mathbb{K}_{a, \alpha, t}$. Moreover by straightforward estimates

$$
\left|\Lambda\left(X^{1}, t\right)-\Lambda\left(X^{2}, t\right)\right|_{\mathbb{K}_{a, \alpha, t}}^{p} \leq g^{p}(a) C_{F}^{p}\left|X^{1}-X^{2}\right|_{\mathbb{K}_{a, \alpha, t}}^{p}
$$

where

$$
g(a)=\sup _{t \in[0, T]} t^{1-\alpha} \int_{0}^{1}(1-s)^{-\alpha} s^{-\alpha} \mathrm{e}^{-a t s} \mathrm{~d} s .
$$

By the Cauchy-Schwartz inequality $g(a) \leq T^{1 / 2-\alpha} a^{-1 / 2}\left(\int_{0}^{1}(1-s)^{-2 \alpha} s^{-2 \alpha} \mathrm{d} s\right)^{1 / 2}$ thus if $a$ is large enough $\Lambda(\cdot, t)$ is a contraction in $\mathbb{K}_{a, \alpha, t}$. The unique fixed point is clearly a mild solution of equation (2.2) and (2.4) holds. Uniqueness is an immediate consequence of the Gronwall lemma. Equation (2.3) can be treated in an identical way.

\subsection{Reformulation of the cost functional}

In this section we assume that Hypothesis 2.2 holds. We define

$$
L(s, x, u)=\int_{0}^{\pi} \ell\left(s, \xi, x(\xi), u^{1}, u^{2}\right) \mathrm{d} \xi, \quad \Phi(x)=\int_{0}^{\pi} \phi(\xi, x(\xi)) \mathrm{d} \xi,
$$

for $s \in[0, T], x=x(\cdot) \in H=L^{2}(0, \pi), u=\left(u^{1} \quad u^{2}\right) \in \mathcal{U}$. The functions $L:[0, T] \times H \times \mathcal{U} \rightarrow \mathbb{R}$ and $\Phi: H \rightarrow \mathbb{R}$ are well defined and measurable. The cost functional (1.3) can be written in the form

$$
J(t, x, u(\cdot))=\mathbb{E} \int_{t}^{T} L\left(s, X_{s}^{u}, u(s)\right) \mathrm{d} s+\mathbb{E} \Phi\left(X_{T}^{u}\right) .
$$

It is easy to show that the cost is finite for any admissible control $u(\cdot)$. Moreover for $s \in[0, T], x \in H, z \in \mathbb{R}^{2}$ we define the hamiltonian:

$$
\Psi(s, x, z)=\inf _{u \in \mathcal{U}}\{z u+L(s, x, u)\},
$$

where $z u$ denotes the scalar product in $\mathbb{R}^{2}$. Since, as it is easy to check, for all $s \in[0, T]$ and all $x \in H, L(s, x, \cdot)$ is continuous on the compact set $\mathcal{U}$ the above infimum is attained. Therefore if we define

$$
\Gamma(s, x, z)=\{u \in \mathcal{U}: z u+L(s, x, u)=\Psi(s, x, z)\}
$$

then $\Gamma(s, x, z) \neq \emptyset$ for every $s \in[0, T]$, every $x \in H$ and every $z \in \mathbb{R}^{2}$. By [5], see Theorems 8.2.10 and 8.2.11, $\Gamma$ admits a measurable selection, i.e. there exists a measurable function $\gamma:[0, T] \times H \times \mathbb{R}^{2} \rightarrow \mathcal{U}$ with $\gamma(s, x, z) \in \Gamma(s, x, z)$ for every $s \in[0, T]$, every $x \in H$ and every $z \in \mathbb{R}^{2}$.

Proposition 2.5. Under Hypothesis 2.2 the following holds.

(1) $\left|\Phi\left(x_{1}\right)-\Phi\left(x_{2}\right)\right|_{H} \leq C_{\phi}\left(1+\left|x_{1}\right|+\left|x_{2}\right|\right)\left|x_{2}-x_{1}\right|$ for all $x_{1}, x_{2}$ in $H$.

(2) There exists a constant $C_{\psi}$ such that $\left|\Psi\left(t, x_{1}, z\right)-\Psi\left(t, x_{2}, z\right)\right| \leq C_{\psi}\left(1+\left|x_{1}\right|+\left|x_{2}\right|\right)\left|x_{2}-x_{1}\right|$ for all $x_{1}$, $x_{2}$ in $H, z \in \mathbb{R}^{2}$ and $t \in[0, T]$.

(3) Setting $C_{\mathcal{U}}=\sup \{|u|: u \in \mathcal{U}\}$ we have $\left|\Psi\left(s, x, z_{1}\right)-\Psi\left(s, x, z_{2}\right)\right| \leq C_{\mathcal{U}}\left|z_{1}-z_{2}\right|$, for every $s \in[0, T]$, $x \in H, z_{1}, z_{2} \in \mathbb{R}^{2}$.

(4) $\sup _{s \in[0, T]}|\Psi(s, 0,0)| \leq C_{\ell}$. 
Proof. Points (1), (2) and (4) are easy to prove. To prove point (3) we note that

$$
z_{1} u+L(s, x, u) \leq c\left|z_{1}-z_{2}\right|+z_{2} u+L(s, x, u)
$$

so that taking the infimum for $u \in \mathcal{U}$ we obtain

$$
\Psi\left(s, x, z_{1}\right) \leq c\left|z_{1}-z_{2}\right|+\Psi\left(s, x, z_{2}\right)
$$

and the proof is completed.

Some of our results are based on the following assumptions:

Hypothesis 2.6. For almost every $\xi \in[0, \pi]$ the map $\phi(\xi, \cdot)$ is continuously differentiable on $\mathbb{R}$. For almost every $s \in[0, T]$ the map $\Psi(s, \cdot, \cdot)$ is Gâteaux differentiable on $H \times \mathbb{R}^{2}$ and the maps $(x, h, z) \rightarrow \nabla_{x} \Psi(s, x, z) h$ and $(x, z, \zeta) \rightarrow \nabla_{z} \Psi(s, x, z) \zeta$ are continuous on $H \times H \times \mathbb{R}^{2}$ and $H \times \mathbb{R}^{2} \times \mathbb{R}^{2}$ respectively.

From this assumption and from Hypothesis 2.2 it follows easily that $\Phi$ is Gâteaux differentiable on $H$ and the map $(x, h) \rightarrow \nabla \Phi(x) h$ is continuous on $H \times H$.

Remark 2.7. From Proposition 2.5 we immediately deduce the following estimates:

$$
|\nabla \Phi(x) h| \leq C_{\phi}(1+2|x|)|h|, \quad\left|\nabla_{x} \Psi(t, x, z) h\right| \leq C_{\psi}(1+2|x|)|h|, \quad\left|\nabla_{z} \Psi(s, x, z) \zeta\right| \leq C_{\mathcal{U}}|\zeta|
$$

Hypothesis 2.6 involves conditions on the function $\Psi$, and not on the function $\ell$ that determines $\Psi$. However, Hypothesis 2.6 can be verified in concrete situations, as the following example shows.

Example 2.7.1. Suppose that the function $\phi$ satisfies the assumptions required in Hypotheses 2.2 and 2.6 . Let $\mathcal{U}$ be the closed ball of radius $R>0$ in $\mathbb{R}^{2}$, centered at the origin. Suppose that the function $\ell$ is defined, for $t \in[0, T], \xi \in[0, \pi], y \in \mathbb{R},\left(u^{1}, u^{2}\right) \in \mathcal{U}$, by

$$
\ell\left(t, \xi, y, u^{1}, u^{2}\right)=\frac{1}{2}|u|^{2}+\ell_{0}(t, \xi, y)
$$

where $\ell_{0}:[0, T] \times[0, \pi] \times \mathbb{R} \rightarrow \mathbb{R}$ is measurable and satisfies

$$
\left|\ell_{0}\left(t, \xi, y_{1}\right)-\ell_{0}\left(t, \xi, y_{2}\right)\right| \leq C_{0}\left(1+\left|y_{1}\right|+\left|y_{2}\right|\right)\left|y_{1}-y_{2}\right|, \quad t \in[0, T], \xi \in[0, \pi], y_{1}, y_{2} \in \mathbb{R}
$$

for some constant $C_{0}$. It follows easily that

$$
\Psi(t, x, z)=\int_{0}^{\pi} \ell_{0}(t, \xi, x(\xi)) \mathrm{d} \xi+g(|z|), \quad t \in[0, T], x \in H, z \in \mathbb{R}^{2},
$$

where $g(r)=-r^{2} / 2$ for $r \leq R$ and $g(r)=-r R+R^{2} / 2$ for $r>R$. If, in addition, we assume that for every $t \in[0, T]$ and $\xi \in[0, \pi]$ the function $\ell_{0}(t, \xi, \cdot): \mathbb{R} \rightarrow \mathbb{R}$ is continuously differentiable then we conclude that all the assumptions in Hypotheses 2.2 and 2.6 are satisfied.

In particular our results cover the case of the quadratic cost (compare with (1.3))

$$
J(t, x, u(\cdot))=\mathbb{E} \int_{t}^{T}\left[|u(s)|^{2}+\int_{0}^{\pi} y(s, \xi)^{2} \mathrm{~d} \xi\right] \mathrm{d} s+\mathbb{E} \int_{0}^{\pi} y(T, \xi)^{2} \mathrm{~d} \xi
$$

with $u(\cdot)$ taking values in a closed ball of $\mathbb{R}^{2}$. 


\section{FurTher PROPERTIES OF THE FORWARD EQUATION}

In this section we consider again the solution of the forward equation (2.3), i.e. of the uncontrolled state equation on the time interval $[t, T]$ with initial condition $x \in H$. It will be denoted by $X(s, t, x)$, to stress dependence on the initial data $t$ and $x$. It is also convenient to extend the process $X(\cdot, t, x)$ letting $X(s, t, x)=x$ for $s \in[0, t]$.

In this section we assume that Hypothesis 2.1 holds, but all the results remain true under the conditions stated in Proposition 2.3.

\subsection{Regular dependence on initial conditions}

Next we consider the dependence of the process $\{X(s, t, x), s \in[0, T]\}$ on the parameters $t, x$.

Proposition 3.1. For any $p \geq 1$ the following holds.

(1) The map $(t, x) \rightarrow X(\cdot, t, x)$ defined on $[0, T] \times H$ and with values in $L_{\mathcal{P}}^{p}(\Omega ; C([0, T] ; H))$ is continuous.

(2) For every $t \in[0, T]$ the map $x \rightarrow X(\cdot, t, x)$ has, at every point $x \in H$, a Gâteaux derivative $\nabla X(\cdot, t, x)$. The map $(t, x, h) \rightarrow \nabla X(\cdot, t, x) h$ is continuous as a map $[0, T] \times H \times H \rightarrow L_{\mathcal{P}}^{p}(\Omega ; C([0, T] ; H))$ and, for every $h \in H$, the following equation holds $\mathbb{P}$-a.s.:

$$
\nabla X(s, t, x) h=\mathrm{e}^{(s-t) A} h+\int_{t}^{s} \mathrm{e}^{(s-\sigma) A} \nabla_{x} F(\sigma, X(\sigma, t, x)) \nabla_{x} X(\sigma, t, x) h \mathrm{~d} \sigma, \quad s \in[t, T],
$$

whereas $\nabla X(s, t, x) h=h$ for $s \in[0, t]$.

Proof. Point 1: continuity.

We slightly modify the definition of the mapping $\Lambda$ introduced above (in the special case $\alpha=0$ ) letting for all $X$ belonging to $L^{p}(\Omega, C([0, T], H))$

$$
\Lambda(X, t, x)(s)= \begin{cases}\int_{t}^{s} \mathrm{e}^{(s-r) A} F\left(r, X_{r}\right) \mathrm{d} r+\mathrm{e}^{(s-t) A} x+V_{1}(s, t) & \text { if } s \geq t \\ x & \text { if } s<t .\end{cases}
$$

In the proof of the previous proposition we have shown that, for $a$ large enough, $\Lambda(\cdot, t, x)$ is a contraction, with contraction constant independent on $t$ and $x$, in the space $L_{\mathcal{P}}^{p}(\Omega ; C([0, T] ; H))$ endowed with the norm

$$
\mathbb{E} \sup _{s \in[0, T]} \mathrm{e}^{\text {pas }}\left|X_{s}\right|_{H}^{p}
$$

equivalent to the natural one. Moreover $X(\cdot, t, x)$ is the unique fixed point of $\Lambda(\cdot, t, x)$.

Thus by parameter dependent contraction argument (see for instance [14], Prop. 2.4) the claim follows if we show that for all $X \in L_{\mathcal{P}}^{p}(\Omega ; C([0, T] ; H))$ the map $(t, x) \rightarrow \Lambda(X, t, x)$ is continuous as a map $[0, T] \times H \rightarrow$ $L_{\mathcal{P}}^{p}(\Omega ; C([0, T] ; H))$.

To prove this, we begin extending $V_{1}(\cdot, t)$ letting $V_{1}(s, t)=0$ for $s \in[0, t]$. We know that $V_{1}(\cdot, t) \in$ $L_{\mathcal{P}}^{p}(\Omega, C([0, T] ; H))$ and we claim that the map $t \rightarrow V_{1}(\cdot, t)$ is continuous with values in $L_{\mathcal{P}}^{p}(\Omega, C([0, T] ; H))$. Fix $t \in[0, T]$ and let $t_{n}^{+} \searrow t, t_{n}^{-} \nearrow t$,

$$
\begin{aligned}
\mathbb{E} \sup _{s \in[0, T]}\left|V_{1}\left(s, t_{n}^{-}\right)-V_{1}\left(s, t_{n}^{+}\right)\right|^{p} & \leq \mathbb{E} \sup _{s \in\left[t_{n}^{-}, T\right]}\left|\int_{t_{n}^{-}}^{s \wedge t_{n}^{+}}(\lambda-A)^{1-\beta} \mathrm{e}^{(s-\sigma)}(\lambda-A)^{\beta} b \mathrm{~d} W_{\sigma}\right|_{H}^{p} \\
& \leq \mathbb{E} \sup _{s \in\left[t_{n}^{-}, t_{n}^{+}\right]}\left|V_{1}\left(s, t_{n}^{-}\right)\right|_{H}^{p} \leq \mathbb{E} \sup _{s \in\left[0, t_{n}^{+}-t_{n}^{-}\right]}\left|V_{1}(s, 0)\right|_{H}^{p} \rightarrow 0
\end{aligned}
$$


where the final convergence comes as an immediate consequence of the dominated convergence theorem since $V_{1}(\cdot, 0) \in L^{p}(\Omega ; C([0, T] ; H))$.

Similarly if we extend $\mathrm{e}^{(s-t) A} x=x$ for $s<t$ then

$$
\sup _{s \in[0, T]}\left|\mathrm{e}^{\left(s-t_{n}^{+}\right) A} x-\mathrm{e}^{\left(s-t_{n}^{-}\right) A} x\right| \rightarrow 0
$$

moreover the map $x \rightarrow \mathrm{e}^{(\cdot-t) A} x$ considered with values in $C([0, T], H)$ is clearly continuous in $x$ uniformly in $t$.

Finally if we let:

$$
V_{3}(s, t)= \begin{cases}\int_{t}^{s} \mathrm{e}^{(s-\sigma) A} F\left(\sigma, X_{\sigma}\right) \mathrm{d} \sigma & \text { if } s \geq t \\ 0 & \text { if } s<t\end{cases}
$$

then considering again the two sequences $t_{n}^{+} \searrow t, t_{n}^{-} \nearrow t$

$$
\begin{aligned}
\mathbb{E}\left(\sup _{s \in[0, T]}\left|V_{3}\left(s, t_{n}^{+}\right)-V_{3}\left(s, t_{n}^{-}\right)\right|_{H}\right)^{p} & \leq \mathbb{E}\left(\sup _{s \in\left[t_{n}^{-}, T\right]}\left|\int_{t_{n}^{-}}^{s \wedge t_{n}^{+}} \mathrm{e}^{(s-\sigma) A} F\left(X_{\sigma}\right) \mathrm{d} \sigma\right|_{H}^{p}\right)^{p} \\
& \leq \mathbb{E}\left(\sup _{s \in\left[t_{n}^{-}, t_{n}^{+}\right]} \int_{t_{n}^{-}}^{s}\left|F\left(X_{\sigma}\right)\right|_{H} \mathrm{~d} \sigma\right)^{p} \leq\left(t_{n}^{+}-t_{n}^{-}\right)^{p} C_{F}^{p} \mathbb{E}\left(1+\sup _{s \in[0, T]}\left|X_{s}\right|\right)^{p} \rightarrow 0 .
\end{aligned}
$$

Point 2: differentiability.

Again by [14], Proposition 2.4, it is enough to show that the map $(X, t, x) \rightarrow \Lambda(X, t, x)$ defined on $L_{\mathcal{P}}^{p}(\Omega, C([0, T] ; H)) \times[0, T] \times H$ with values in $L_{\mathcal{P}}^{p}(\Omega, C([0, T] ; H))$ is Gâteaux differentiable in $(X, x)$ and has strongly continuous derivatives.

Under our assumptions the Gâteaux derivatives clearly exist and have the simple form:

$$
\begin{aligned}
& \nabla_{X} \Lambda(X, t, x) N=\left\{\begin{array}{ll}
\int_{t}^{s} \mathrm{e}^{(s-\sigma) A} \nabla_{x} F\left(\sigma, X_{\sigma}\right) N_{\sigma} \mathrm{d} \sigma & \text { if } s \geq t, \\
0 & \text { if } s<t,
\end{array} \quad N \in L_{\mathcal{P}}^{p}(\Omega, C([0, T] ; H)) .\right. \\
& \nabla_{x} \Lambda(X, t, x) h=\left\{\begin{array}{ll}
\mathrm{e}^{(s-t) A} h & \text { if } s \geq t, \\
h & \text { if } s<t,
\end{array} \quad h \in H .\right.
\end{aligned}
$$

Notice that the above maps are considered as maps having values in $L_{\mathcal{P}}^{p}(\Omega, C([0, T] ; H))$.

The continuity of $\nabla_{x} \Lambda(X, t, x) h$ in all variables is immediate. Moreover $\nabla_{X} \Lambda(X, t, x)$ is a bounded linear operator in $L_{\mathcal{P}}^{p}(\Omega, C([0, T] ; H))$ uniformly in $X$ and $t$. Thus it is enough to show that for all fixed $N \in$ $L_{\mathcal{P}}^{p}(\Omega, C([0, T] ; H))$ the map $(t, X) \rightarrow \nabla_{X} \Lambda(X, t, x) N$ is continuous. The continuity in $t$ for $X$ fixed can be proved exactly as we have done to prove continuity in $t$ of $\Lambda(X, t, x)$. It remains to show that the map $X \rightarrow \nabla_{X} \Lambda(X, t, x) N$ is continuous uniformly in $t$. Assume that this is not true; then we can find a sequence $X^{n} \rightarrow X$ in $L_{\mathcal{P}}^{p}(\Omega, C([0, T] ; H))$ for which

$$
\lim _{n} \sup _{t \in[0, T]}\left|\nabla_{X} \Lambda\left(X^{n}, t, x\right) N-\Lambda(X, t, x) N\right|_{L_{\mathcal{P}}^{p}(\Omega, C([0, T] ; H))} \neq 0
$$


Clearly we can always assume that $X_{\sigma}^{n} \rightarrow X_{\sigma}$ for all $\sigma \in[0, T], \mathbb{P}$-a.s.. Then

$$
\begin{aligned}
\sup _{t \in[0, T]} \mid \nabla_{X} \Lambda\left(X^{n}, t, x\right) N & -\left.\Lambda(X, t, x) N\right|_{L_{\mathcal{P}}^{p}(\Omega, C([0, T] ; H))} \\
& \leq \mathbb{E} \sup _{0 \leq t \leq s \leq T}\left|\int_{t}^{s} \mathrm{e}^{(s-\sigma) A}\left(\nabla_{x} F\left(\sigma, X_{\sigma}^{n}\right)-\nabla_{x} F\left(\sigma, X_{\sigma}\right)\right) N_{\sigma} \mathrm{d} \sigma\right|^{p} \\
& \leq \mathbb{E} T^{p-1} \int_{0}^{T}\left|\left(\nabla_{x} F\left(\sigma, X_{\sigma}^{n}\right)-\nabla_{x} F\left(\sigma, X_{\sigma}\right)\right) N_{\sigma}\right|^{p} \mathrm{~d} \sigma \rightarrow 0 .
\end{aligned}
$$

where the last convergence is a direct consequence of the dominated convergence theorem.

The claim of Point 2 now follows directly by [14], Proposition 2.4.

Proposition 3.2. For every $\alpha \in[0,1)$ there exists a family of predictable processes $\left\{\Theta^{\alpha}(\cdot, t, x) h: h \in H, x \in\right.$ $H, t \in[0, T]\}$ all defined on $\Omega \times[0, T] \rightarrow H$ such that the following holds:

(1) The map $h \rightarrow \Theta^{\alpha}(\cdot, t, x) h$ is linear and, if $h \in D(\lambda-A)^{\alpha}$, then

$$
\Theta^{\alpha}(s, t, x) h= \begin{cases}\left(\nabla_{x} X(s, t, x)-\mathrm{e}^{(s-t) A}\right)(\lambda-A)^{\alpha} h & \text { if } s \in[t, T], \\ 0 & \text { if } s \in[0, t) .\end{cases}
$$

(2) The map $(t, x, h) \rightarrow \Theta^{\alpha}(\cdot, t, x) h$ is continuous $[0, T] \times H \times H \rightarrow L_{\mathcal{P}}^{\infty}(\Omega ; C([0, T] ; H))$.

(3) There exists a constant $C_{\theta, \alpha}$ such that

$$
\left|\Theta^{\alpha}(\cdot, t, x) h\right|_{L_{\mathcal{P}}^{\infty}(\Omega, C([0, T] ; H))} \leq C_{\theta, \alpha}|h| \text { for all } t \in[0, T], x, h \in H .
$$

Proof. For fixed $t \in[0, T]$ and $x, h \in H$ consider the equation:

$$
\begin{aligned}
\Theta^{\alpha}(s, t, x) h= & \int_{t}^{s} \mathrm{e}^{(s-\sigma) A} \nabla_{x} F(\sigma, X(\sigma, t, x)) \Theta^{\alpha}(\sigma, t, x) h \mathrm{~d} \sigma \\
& +\int_{t}^{s} \mathrm{e}^{(s-\sigma) A} \nabla_{x} F(\sigma, X(\sigma, t, x))(\lambda-A)^{\alpha} \mathrm{e}^{(\sigma-t) A} h \mathrm{~d} \sigma .
\end{aligned}
$$

Notice that

$$
\int_{t}^{s}\left|\mathrm{e}^{(s-\sigma) A} \nabla_{x} F(\sigma, X(\sigma, t, x))(\lambda-A)^{\alpha} \mathrm{e}^{(\sigma-t) A} h\right| \mathrm{d} \sigma \leq C_{F} \int_{t}^{s}(\sigma-t)^{-\alpha}|h| \mathrm{d} \sigma \leq c|h|
$$

for a suitable constant $c$.

Thus being $\nabla_{x} F$ bounded it is immediate to prove that equation (3.6) has $\mathbb{P}$-almost surely a unique solution in $C([t, T] ; H)$. Moreover extending $\Theta^{\alpha}(s, t, x) h=0$ for $s<t$ and considering it as a process we have $\Theta(\cdot, t, x) h \in$ $L_{\mathcal{P}}^{\infty}(\Omega, C([0, T] ; H))$ and $\left|\Theta^{\alpha}(\cdot, t, x) h\right|_{L_{\mathcal{P}}^{\infty}(\Omega, C([0, T] ; H))} \leq C_{\alpha}|k|$. The continuity with respect to $t$ and $x$ and $k$ can easily be shown by parameter dependent contraction argument as in the proof of the previous Proposition. Moreover linearity in $h$ is straight-forward. Finally for all $k \in D(-A)^{\alpha}$ setting $h=(\lambda-A)^{\alpha} k$ equation $(3.1)$ can be rewritten:

$$
\begin{aligned}
& \left(\nabla_{x} X(s, t, x)(\lambda-A)^{\alpha} k-\mathrm{e}^{(s-t) A}(\lambda-A)^{\alpha} k\right)=\int_{t}^{s} \mathrm{e}^{(s-\sigma) A} \nabla_{x} F(\sigma, X(\sigma, t, x)) \mathrm{e}^{(\sigma-t) A}(\lambda-A)^{\alpha} k \mathrm{~d} \sigma \\
& \quad+\int_{t}^{s} \mathrm{e}^{(s-\sigma) A} \nabla_{x} F(\sigma, X(\sigma, t, x))\left(\nabla_{x} X(\sigma, t, x)(\lambda-A)^{\alpha} k-\mathrm{e}^{(\sigma-t) A}(\lambda-A)^{\alpha} k\right) \mathrm{d} \sigma .
\end{aligned}
$$

Comparing the above equation with equation (3.6) by a straightforward application of Gronwall Lemma get $\Theta^{\alpha}(s, t, x) k=\left(\nabla_{x} X(s, t, x)-\mathrm{e}^{(s-t) A}\right)(\lambda-A)^{\alpha} k \mathbb{P}$-a.s. for all $s \in[t, T]$. 
Remark 3.3. In the special case $\alpha=0$ relation (3.5) yields that there exists $C_{\nabla X}$

$$
\left|\nabla_{x} X(\cdot, t, x) h\right|_{L_{\mathcal{P}}^{\infty}(\Omega, C([0, T] ; H))} \leq C_{\nabla X}|h| \text { for all } t \in[0, T], x, h \in H
$$

and consequently, for all $p \in[1,+\infty)$ there exists a constant $C_{X, p}$ such that:

$$
|X(\cdot, t, x)|_{L_{\mathcal{P}}^{p}(\Omega, C([0, T] ; H))} \leq C_{X, p}\left(1+|x|_{H}\right) \text { for all } t \in[0, T], x \in H .
$$

\subsection{Regularity in the Malliavin sense}

In order to state the following results we need to recall some basic definitions from the Malliavin calculus, mainly to fix notation. We refer the reader to the book [24] for a detailed exposition; the paper [20] treats the extensions to Hilbert space valued random variables and processes.

For every $h \in L^{2}\left([0, T] ; \mathbb{R}^{2}\right)$ we denote by $h^{i} \in L^{2}(0, T), i=1,2$, its components and use the notation

$$
W(h)=\int_{0}^{T} h(t) \mathrm{d} W(t)=\int_{0}^{T} h^{1}(t) \mathrm{d} W^{1}(t)+\int_{0}^{T} h^{2}(t) \mathrm{d} W^{2}(t) .
$$

Given a Hilbert space $K$, let $S_{K}$ be the set of $K$-valued random variables $F$ of the form

$$
F=\sum_{j=1}^{m} f_{j}\left(W\left(h_{1}\right), \ldots, W\left(h_{n}\right)\right) e_{j}
$$

where $h_{1}, \ldots, h_{n} \in L^{2}\left([0, T] ; \mathbb{R}^{2}\right),\left\{e_{j}\right\}$ is a basis of $K$ and $f_{1}, \ldots, f_{m}$ are infinitely differentiable functions $\mathbb{R}^{n} \rightarrow \mathbb{R}$ bounded together with all their derivatives. The Malliavin derivative $D F$ of $F \in S_{K}$ is defined as the process $D_{s} F=\left(D_{s}^{1} F \quad D_{s}^{2} F\right), s \in[0, T]$, where

$$
D_{s}^{i} F=\sum_{j=1}^{m} \sum_{k=1}^{n} \partial_{k} f_{j}\left(W\left(h_{1}\right), \ldots, W\left(h_{n}\right)\right) h_{k}^{i}(s) e_{j}, \quad i=1,2 .
$$

And by $\partial_{k}$ we denote the partial derivative with respect to the $k$-th variable. Thus $D_{s}^{i} F(\omega)$ is an element of $K$ and $D F$ is a process with values in $K^{2}:=K \times K$, that we will identify with an element of $L^{2}\left(\Omega \times[0, T] ; K^{2}\right)$ with the corresponding norm:

$$
\|D F\|_{L^{2}\left(\Omega \times[0, T] ; K^{2}\right)}^{2}=\mathbb{E} \sum_{i=1}^{2} \int_{0}^{T}\left\|D_{s}^{i} F\right\|_{K}^{2} \mathrm{~d} s .
$$

It is known that the operator $D: S_{K} \subset L^{2}(\Omega ; K) \rightarrow L^{2}\left(\Omega \times[0, T] ; K^{2}\right)$ is closable. We denote by $\mathbb{D}^{1,2}(K)$ the domain of its closure, and use the same letter to denote $D$ and its closure:

$$
D: \mathbb{D}^{1,2}(K) \subset L^{2}(\Omega ; K) \rightarrow L^{2}\left(\Omega \times[0, T] ; K^{2}\right) .
$$

The adjoint operator of $D$,

$$
\delta: \operatorname{dom}(\delta) \subset L^{2}\left(\Omega \times[0, T] ; K^{2}\right) \rightarrow L^{2}(\Omega ; K),
$$

is called Skorohod integral. It is known that $\operatorname{dom}(\delta)$ contains $L_{\mathcal{P}}^{2}\left(\Omega ; L^{2}\left([0, T] ; K^{2}\right)\right)$ and the Skorohod integral of a process in this space coincides with the Itô integral. The class $\mathbb{L}^{1,2}\left(K^{2}\right)$ is also contained in $\operatorname{dom}(\delta)$, the latter being defined as the space of processes $u \in L^{2}\left(\Omega \times[0, T] ; K^{2}\right)$ such that $u_{r} \in \mathbb{D}^{1,2}\left(K^{2}\right)$ for a.e. $r \in[0, T]$ and there exists a measurable version of $D_{s} u_{r}$ satisfying

$$
\|u\|_{\mathbb{L}^{1,2}\left(K^{2}\right)}^{2}=\|u\|_{L^{2}\left(\Omega \times[0, T] ; K^{2}\right)}^{2}+\mathbb{E} \sum_{i=1}^{2} \int_{0}^{T} \int_{0}^{T}\left\|D_{s}^{i} u_{r}\right\|_{K^{2}}^{2} \mathrm{~d} r \mathrm{~d} s<\infty .
$$


Moreover, $\|\delta(u)\|_{L^{2}(\Omega ; K)}^{2} \leq\|u\|_{\mathbb{L}^{1,2}\left(K^{2}\right)}^{2}$. The definition of $\mathbb{L}^{1,2}(K)$ for an arbitrary Hilbert space $K$ is entirely analogous; clearly, $\mathbb{L}^{1,2}(K)$ is isomorphic to $L^{2}\left([0, T] ; \mathbb{D}^{1,2}(K)\right)$.

Finally we recall that if $F \in \mathbb{D}^{1,2}(K)$ is $\mathcal{F}_{t}$-adapted then $D F=0$ a.s. on $\Omega \times(t, T]$.

Now for $(t, x)$ fixed let us consider again the process $\{X(s, t, x), s \in[t, T]\}$ solution of the forward equation (2.3). It will be denoted simply by $\left\{X_{s}, s \in[t, T]\right\}$ or even $X$. We still agree that $X_{s}=x$ for $s \in[0, t)$. We will soon prove that $X$ belongs to $\mathbb{L}^{1,2}(H)$. Then it is clear that the equality $D_{\sigma} X_{s}=0 \mathbb{P}$-a.s. holds for a.a. $\sigma, t, s$ if $s<t$ or $\sigma>s$.

In the rest of this section we still assume that Hypothesis 2.1 holds.

Proposition 3.4. Let $t \in[0, T]$ and $x \in H$ be fixed. Then $X \in \mathbb{L}^{1,2}(H)$, and $\mathbb{P}$-a.s. we have, for a.a. $\sigma, s$ such that $t \leq \sigma \leq s \leq T$,

$$
\begin{gathered}
D_{\sigma} X_{s}=(\lambda-A) \mathrm{e}^{(s-\sigma) A} b+\int_{\sigma}^{s} \mathrm{e}^{(s-r) A} \nabla F\left(r, X_{r}\right) D_{\sigma} X_{r} \mathrm{~d} r, \\
\left|D_{\sigma} X_{s}\right| \leq C(s-\sigma)^{\beta-1} .
\end{gathered}
$$

Moreover for every $s \in[0, T]$ we have $X_{s} \in \mathbb{D}^{1,2}(H)$ and $D X_{s} \in L^{\infty}\left(\Omega ; L^{2}\left([0, T] ; H^{2}\right)\right)$.

Finally, for every $q \in[2, \infty)$ the map $s \rightarrow X_{s}$ is continuous from $[0, T]$ to $L^{q}(\Omega ; H)$ and the map $s \rightarrow D X_{s}$ is continuous from $[0, T]$ to $L^{q}\left(\Omega ; L^{2}\left([0, T] ; H^{2}\right)\right)$.

Proof. For simplicity of notation we write the proof for the case $t=0$. Thus,

$$
X_{s}=\mathrm{e}^{s A} x+\int_{0}^{s} \mathrm{e}^{(s-r) A} F\left(r, X_{r}\right) \mathrm{d} r+\int_{0}^{s}(\lambda-A)^{1-\beta} \mathrm{e}^{(s-r) A}(\lambda-A)^{\beta} b \mathrm{~d} W_{r} \quad s \in[0, T] .
$$

We set $J_{n}=n(n-A)^{-1}$ and we consider the approximating equation

$$
\begin{aligned}
X_{s}^{n} & =\mathrm{e}^{s A} x+\int_{0}^{s} \mathrm{e}^{(s-r) A} F\left(r, X_{r}^{n}\right) \mathrm{d} r+\int_{0}^{s}(\lambda-A)^{1-\beta} \mathrm{e}^{(s-r) A}(\lambda-A)^{\beta} J_{n} b \mathrm{~d} W_{r}, \\
& =\mathrm{e}^{s A} x+\int_{0}^{s} \mathrm{e}^{(s-r) A} F\left(r, X_{r}^{n}\right) \mathrm{d} r+\int_{0}^{s} \mathrm{e}^{(s-r) A}(\lambda-A) J_{n} b \mathrm{~d} W_{r}, \quad s \in[0, T] .
\end{aligned}
$$

Since $(\lambda-A) J_{n}$ is a linear bounded operator in $H$, we can apply Proposition 3.5 of [14] and conclude that $X^{n} \in \mathbb{L}^{1,2}(H)$, and that $\mathbb{P}$-a.s. we have, for a.a. $\sigma, s$ such that $0 \leq \sigma \leq s \leq T$,

$$
D_{\sigma} X_{s}^{n}=(\lambda-A) \mathrm{e}^{(s-\sigma) A} J_{n} b+\int_{\sigma}^{s} \mathrm{e}^{(s-r) A} \nabla F\left(r, X_{r}^{n}\right) D_{\sigma} X_{r}^{n} \mathrm{~d} r .
$$

Since

$$
\left|(\lambda-A) \mathrm{e}^{(s-\sigma) A} J_{n} b\right| \leq\left|(\lambda-A)^{1-\beta} \mathrm{e}^{(s-r) A}\right|\left|J_{n}\right|\left|(\lambda-A)^{\beta} b\right| \leq C(s-r)^{\beta-1},
$$

by the boundedness of $\nabla F$ and the Gronwall Lemma it is easy to deduce that $\left|D_{\sigma} X_{s}^{n}\right| \leq C(s-\sigma)^{\beta-1}$. In particular it follows that $D X^{n}$ is bounded in the space $L^{2}\left(\Omega \times[0, T] \times[0, T] ; H^{2}\right)$.

Subtracting (3.12) from (3.11) and using the Lipschitz character of $F$ we obtain

$$
\mathbb{E}\left|X_{s}^{n}-X_{s}\right|^{2} \leq C \int_{0}^{s} \mathbb{E}\left|X_{r}^{n}-X_{r}\right|^{2} \mathrm{~d} r+C \int_{0}^{s}\left|(\lambda-A)^{1-\beta} \mathrm{e}^{(s-r) A}(\lambda-A)^{\beta}\left(J_{n} b-b\right)\right|^{2} \mathrm{~d} r .
$$

The last integral can be estimated by

$$
C \int_{0}^{s}(s-r)^{2 \beta-2} \mathrm{~d} r\left|\left(J_{n}-I\right)(\lambda-A)^{\beta} b\right|^{2} \leq C\left|\left(J_{n}-I\right)(\lambda-A)^{\beta} b\right|^{2},
$$

which tends to zero, by well-known properties of the operators $J_{n}$. If follows from the Gronwall Lemma that $\sup _{s} \mathbb{E}\left|X_{s}^{n}-X_{s}\right|^{2} \rightarrow 0$ and in particular $X^{n} \rightarrow X$ in $L^{2}(\Omega \times[0, T] ; H)$. 
The boundedness of the sequence $D X^{n}$ proved before and the closedness of the operator $D$ imply that $X \in \mathbb{L}^{1,2}(H)$ and that $D X^{n} \rightarrow D X$ weakly in the space $L^{2}\left(\Omega \times[0, T] \times[0, T] ; H^{2}\right)$. Passing to the limit in (3.13) is easily justified and this proves equation (3.9). The estimate (3.10) on $D X$ can be proved in the same way as it was done for $D X^{n}$.

We note that for any fixed $s \in[0, T]$, the estimate $\left|D_{\sigma} X_{s}^{n}\right| \leq C(s-\sigma)^{\beta-1}$ also shows that $D X_{s}^{n}$ is bounded in the space $L^{2}\left(\Omega \times[0, T] ; H^{2}\right)$. Arguing as before we conclude that $X_{s} \in \mathbb{D}^{1,2}(H)$ for every $s$. The estimate (3.10) implies that $D X_{s} \in L^{\infty}\left(\Omega ; L^{2}\left([0, T] ; H^{2}\right)\right)$.

It remains to prove the continuity statements. The continuity of the map $s \rightarrow X_{s}$ with values in $L^{q}(\Omega ; H)$ follows immediately from Proposition 2.4. To prove the continuity of $s \rightarrow D X_{s}$ as a map $[0, T] \rightarrow L^{q}\left(\Omega ; L^{2}([0, T]\right.$; $\left.\left.H^{2}\right)\right)$ we fix $s \in[0, T]$ and let $s_{n}^{+} \searrow s, s_{n}^{-} \nearrow s$. Then from (3.9) we obtain

$$
\begin{aligned}
\int_{0}^{T} \mid D_{\sigma} X_{s_{n}^{+}}- & \left.D_{\sigma} X_{s_{n}^{-}}\right|^{2} \mathrm{~d} \sigma \leq 2 \int_{0}^{T}\left|(\lambda-A)\left(\mathrm{e}^{\left(s_{n}^{+}-\sigma\right) A} 1_{\sigma<s_{n}^{+}}-\mathrm{e}^{\left(s_{n}^{-}-\sigma\right) A} 1_{\sigma<s_{n}^{-}}\right) b\right|^{2} \mathrm{~d} \sigma \\
& +2 \int_{0}^{T}\left|\int_{\sigma}^{s_{n}^{+}} \mathrm{e}^{\left(s_{n}^{+}-r\right) A} \nabla F\left(r, X_{r}\right) D_{\sigma} X_{r} \mathrm{~d} r-\int_{\sigma}^{s_{n}^{-}} \mathrm{e}^{\left(s_{n}^{-}-r\right) A} \nabla F\left(r, X_{r}\right) D_{\sigma} X_{r} \mathrm{~d} r\right|^{2} \mathrm{~d} \sigma=: I_{1}+I_{2} .
\end{aligned}
$$

Clearly, $I_{1}$ is deterministic and we have

$$
\begin{aligned}
I_{1} & =2 \int_{s_{n}^{-}}^{s_{n}^{+}}\left|(\lambda-A) \mathrm{e}^{\left(s_{n}^{+}-\sigma\right) A} b\right|^{2} \mathrm{~d} \sigma+2 \int_{0}^{s_{n}^{-}}\left|(\lambda-A)\left(\mathrm{e}^{\left(s_{n}^{+}-s_{n}^{-}\right) A}-I\right) \mathrm{e}^{\left(s_{n}^{-}-\sigma\right) A} b\right|^{2} \mathrm{~d} \sigma \\
& \leq C \int_{s_{n}^{-}}^{s_{n}^{+}}\left(s_{n}^{+}-\sigma\right)^{2 \beta-2} \mathrm{~d} \sigma\left|(\lambda-A)^{\beta} b\right|^{2}+C \int_{0}^{s_{n}^{-}}\left(s_{n}^{-}-\sigma\right)^{2 \beta-2} \mathrm{~d} \sigma\left|\left(\mathrm{e}^{\left(s_{n}^{+}-s_{n}^{-}\right) A}-I\right)(\lambda-A)^{\beta} b\right|^{2}
\end{aligned}
$$

which shows that $I_{1} \rightarrow 0$ as $n \rightarrow \infty$. In a similar way, using (3.10) and the dominated convergence theorem, one can show that $\mathbb{E}\left|I_{2}\right|^{q} \rightarrow 0$ and the required continuity follows.

We still set $X_{s}=X(s, 0, x)$, for simplicity. Given a function $w:[0, T] \times H \rightarrow \mathbb{R}$, we investigate the existence of the joint quadratic variation of the process $\left\{w\left(s, X_{s}\right), s \in[0, T]\right\}$ with $W^{j}, j=1,2$, on an interval $[0, s] \subset[0, T)$. As usual, this is defined as the limit in probability of

$$
\left.\sum_{i=1}^{n}\left(w\left(s_{i}, X_{s_{i}}\right)\right)-w\left(s_{i-1}, X_{s_{i-1}}\right)\right)\left(W_{s_{i}}^{j}-W_{s_{i-1}}^{j}\right)
$$

where $\left\{s_{i}\right\}, 0=s_{0}<s_{1}<\cdots<s_{n}=s$ is an arbitrary subdivision of $[0, s]$ whose mesh tends to 0 . We do not require that convergence takes place uniformly in time. This definition is easily adapted to arbitrary interval of the form $[t, s] \subset[0, T)$. Existence of the joint quadratic variation is not trivial. Indeed, due to the occurrence of convolution type integrals in the definition of mild solution, it is not obvious that the process $X$ is a semimartingale. Moreover, even in this case, the process $w(\cdot, X)$ might fail to be a semimartingale if $w$ is not twice differentiable, since the Ito formula does not apply. Nevertheless, the following result holds true. Its proof could be deduced from generalization of some results obtained in [25] to the infinite-dimensional case, but we prefer to give a simpler direct proof.

Proposition 3.5. Suppose that $w \in C([0, T) \times H ; \mathbb{R})$ is Gâteaux differentiable with respect to $x$, and that for every $s<T$ there exist constants $K$ and $m$ (possibly depending on $s$ ) such that

$$
|w(t, x)| \leq K(1+|x|)^{m}, \quad|\nabla w(t, x)| \leq K(1+|x|)^{m}, \quad t \in[0, s], x \in H .
$$

Assume that for every $t \in[0, T), x \in H$ the linear operator $k \rightarrow \nabla w(t, x)(\lambda-A)^{1-\beta} k$ (a priori defined for $\left.k \in D(-A)^{1-\beta}\right)$ has an extension to a bounded linear operator $H \rightarrow \mathbb{R}$, that we denote by $\left[\nabla w(\lambda-A)^{1-\beta}\right](t, x)$. Moreover assume that the map $(t, x, k) \rightarrow\left[\nabla w(\lambda-A)^{1-\beta}\right](t, x) k$ is continuous $[0, T) \times H \times H \rightarrow \mathbb{R}$. 
For $t \in[0, T), x \in H$, let $\{X(s, t, x), s \in[t, T]\}$ be the solution of equation (2.3). Then the process $\{w(s, X(s, t, x)), s \in[t, T]\}$ admits a joint quadratic variation process with $W^{j}, j=1,2$, on every interval $[t, s] \subset[t, T)$, given by

$$
\int_{t}^{s}\left[\nabla w(\lambda-A)^{1-\beta}\right](r, X(r, t, x))(\lambda-A)^{\beta} b^{j} \mathrm{~d} r
$$

Proof. For simplicity we write the proof for the case $t=0$, and we write $X_{s}=X(s, 0, x), w_{s}=w\left(s, X_{s}\right)$. It follows from the assumptions that the map $(t, x, h) \rightarrow \nabla w(t, x) h$ is also continuous on $[0, T) \times H \times H$. By the chain rule for the Malliavin derivative operator (see [14] for details), it follows that for every $s<T$ we have $w_{s} \in \mathbb{D}^{1,2}(\mathbb{R})$ and $D w_{s}=\nabla w\left(s, X_{s}\right) D X_{s}$.

Let us now compute the joint quadratic variation of $w$ and $W^{j}$ on a fixed interval $[0, s] \subset[0, T)$. Let $0=s_{0}<s_{1}<\cdots<s_{n}=s$ be a subdivision of $[0, s] \subset[0, T]$ with mesh $\delta=\max _{i}\left(s_{i}-s_{i-1}\right)$. By well-known rules of Malliavin calculus (see [25], Th. 3.2, or [20], Prop. 2.11) we have

$$
\left(w_{s_{i}}-w_{s_{i-1}}\right)\left(W_{s_{i}}^{j}-W_{s_{i-1}}^{j}\right)=\left(w_{s_{i}}-w_{s_{i-1}}\right) \int_{s_{i-1}}^{s_{i}} \mathrm{~d} W_{\sigma}^{j}=\int_{s_{i-1}}^{s_{i}} D_{\sigma}^{j}\left(w_{s_{i}}-w_{s_{i-1}}\right) \mathrm{d} \sigma+\int_{s_{i-1}}^{s_{i}}\left(w_{s_{i}}-w_{s_{i-1}}\right) \hat{\mathrm{d}} W_{\sigma}^{j},
$$

where we use the symbol $\hat{\mathrm{d}} W$ to denote the Skorohod integral. We note that $D_{\sigma} w_{s_{i-1}}=0$ for $\sigma>s_{i-1}$. Therefore setting $U_{\delta}(\sigma)=\sum_{i=1}^{n}\left(w_{s_{i}}-w_{s_{i-1}}\right) 1_{\left(s_{i-1}, s_{i}\right]}(\sigma)$ we obtain

$$
\sum_{i=1}^{n}\left(w_{s_{i}}-w_{s_{i-1}}\right)\left(W_{s_{i}}^{j}-W_{s_{i-1}}^{j}\right)=\int_{0}^{s} U_{\delta}(\sigma) \hat{\mathrm{d}} W_{\sigma}^{j}+\sum_{i=1}^{n} \int_{s_{i-1}}^{s_{i}} \nabla w\left(s_{i}, X_{s_{i}}\right) D_{\sigma}^{j} X_{s_{i}} \mathrm{~d} \sigma .
$$

Recalling (3.9) we obtain

$$
\begin{aligned}
\sum_{i=1}^{n}\left(w_{s_{i}}-w_{s_{i-1}}\right)\left(W_{s_{i}}^{j}-W_{s_{i-1}}^{j}\right)= & \int_{0}^{s} U_{\delta}(\sigma) \hat{\mathrm{d}} W_{\sigma}^{j}+\sum_{i=1}^{n} \int_{s_{i-1}}^{s_{i}} \nabla w\left(s_{i}, X_{s_{i}}\right)(\lambda-A) \mathrm{e}^{\left(s_{i}-\sigma\right) A} b^{j} \mathrm{~d} \sigma \\
& +\sum_{i=1}^{n} \int_{s_{i-1}}^{s_{i}} \nabla w\left(s_{i}, X_{s_{i}}\right) \int_{\sigma}^{s_{i}} \mathrm{e}^{\left(s_{i}-r\right) A} \nabla F\left(r, X_{r}\right) D_{\sigma} X_{r} \mathrm{~d} r \mathrm{~d} \sigma=: I_{1}+I_{2}+I_{3} .
\end{aligned}
$$

Now we let the mesh $\delta$ tend to 0 .

Using the continuity properties of the maps $s \rightarrow X_{s}$ and $s \rightarrow D X_{s}$ stated in Proposition 3.4, taking into account the continuity properties of $w$ and $\nabla w$, the estimate (3.14) and the chain rule $D w_{s}=\nabla w\left(s, X_{s}\right) D X_{s}$, it is easy to show that the map $s \rightarrow w_{s}=w\left(s, X_{s}\right)$ is continuous from $[0, T]$ to $\mathbb{D}^{1,2}(\mathbb{R})$. It follows that $U_{\delta} \rightarrow 0$ in $\mathbb{L}^{1,2}(\mathbb{R})=L^{2}\left([0, T] ; \mathbb{D}^{1,2}(\mathbb{R})\right)$ and by the continuity of the Skorohod integral we conclude that $I_{1} \rightarrow 0$ in $L^{2}(\Omega ; \mathbb{R})$.

According to the definition of the function $\left[\nabla w(\lambda-A)^{1-\beta}\right]$ the second term can be written

$$
\begin{aligned}
I_{2}= & \sum_{i=1}^{n}\left[\nabla w(\lambda-A)^{1-\beta}\right]\left(s_{i}, X_{s_{i}}\right) \int_{s_{i-1}}^{s_{i}} \mathrm{e}^{\left(s_{i}-\sigma\right) A}(\lambda-A)^{\beta} b^{j} \mathrm{~d} \sigma \\
= & \sum_{i=1}^{n}\left[\nabla w(\lambda-A)^{1-\beta}\right]\left(s_{i}, X_{s_{i}}\right)(\lambda-A)^{\beta} b^{j}\left(s_{i}-s_{i-1}\right) \\
& +\sum_{i=1}^{n}\left[\nabla w(\lambda-A)^{1-\beta}\right]\left(s_{i}, X_{s_{i}}\right) \int_{s_{i-1}}^{s_{i}}\left(\mathrm{e}^{\left(s_{i}-\sigma\right) A}-I\right)(\lambda-A)^{\beta} b^{j} \mathrm{~d} \sigma .
\end{aligned}
$$

We note that for every $i$,

$$
\sup _{\sigma \in\left[s_{i-1}, s_{i}\right]}\left|\left(\mathrm{e}^{\left(s_{i}-\sigma\right) A}-I\right)(\lambda-A)^{\beta} b^{j}\right| \leq \sup _{\sigma \in[0, \delta]}\left|\left(\mathrm{e}^{\sigma A}-I\right)(\lambda-A)^{\beta} b^{j}\right| \rightarrow 0,
$$


as $\delta \rightarrow 0$, by the strong continuity of the semigroup. From the continuity properties of $\left[\nabla w(\lambda-A)^{1-\beta}\right]$ and the continuity of the paths of $X$ it follows that

$$
I_{2} \rightarrow \int_{0}^{s}\left[\nabla w(\lambda-A)^{1-\beta}\right]\left(r, X_{r}\right)(\lambda-A)^{\beta} b^{j} \mathrm{~d} r, \quad \mathbb{P}-a . s .
$$

Using the estimate (3.10) and the boundedness of $\nabla F$ we obtain

$$
\left|I_{3}\right| \leq C \sum_{i=1}^{n}\left|\nabla w\left(s_{i}, X_{s_{i}}\right)\right| \int_{s_{i-1}}^{s_{i}} \int_{\sigma}^{s_{i}}(r-\sigma)^{\beta-1} \mathrm{~d} r \mathrm{~d} \sigma \leq C \sum_{i=1}^{n}\left|\nabla w\left(s_{i}, X_{s_{i}}\right)\right|\left(s_{i}-s_{i-1}\right) \delta^{\beta} .
$$

It follows that $I_{3} \rightarrow 0, \mathbb{P}$-a.s., by the continuity properties of $\nabla w$ and the continuity of the paths of $X$.

\section{The BACKWARD STOCHASTIC DIFFERENTIAL EQUATION}

We consider the following backward stochastic differential equation:

$$
\left\{\begin{array}{l}
\mathrm{d} Y_{s}=\Psi\left(s, X(s, t, x), Z_{s}\right) \mathrm{d} s+Z_{s} \mathrm{~d} W_{s}, \quad s \in[0, T] \\
Y_{T}=\Phi(X(T, t, x))
\end{array}\right.
$$

for unknown processes $Y, Z$ in $\mathbb{R}$ and $\mathbb{R}^{2}$ respectively. The equation is understood in the usual way: $\mathbb{P}$-a.s.,

$$
Y_{s}+\int_{s}^{T} Z_{r} \mathrm{~d} W_{r}=\Phi(X(T, t, x))-\int_{s}^{T} \Psi\left(r, X(r, t, x), Z_{r}\right) \mathrm{d} r, \quad s \in[0, T],
$$

but we will use the shortened notation above for equation (4.1) and similar equations to follow. In (4.1) and (4.2), $t \in[0, T]$ and $x \in H$ are given and the process $X(\cdot, t, x)$ is the solution of $(2.3)$, with the convention that $X(s, t, x)=x$ for $s \in[0, t)$.

\section{Proposition 4.1.}

(1) For all $x \in H, t \in[0, T]$ and $p \in[2, \infty)$ there exists a unique pair of processes $(Y, Z)$ with $Y \in$ $L_{\mathcal{P}}^{p}(\Omega, C([0, T], \mathbb{R})), Z \in L_{\mathcal{P}}^{p}\left(\Omega, L^{2}\left([0, T], \mathbb{R}^{2}\right)\right)$ solving $(4.1) ;$ in the following we denote such a solution by $(Y(\cdot, t, x), Z(\cdot, t, x))$.

(2) The map $(t, x) \rightarrow(Y(\cdot, t, x), Z(\cdot, t, x))$ is continuous from $[0, T] \times H$ to $L_{\mathcal{P}}^{p}(\Omega, C([0, T], \mathbb{R})) \times L_{\mathcal{P}}^{p}(\Omega$, $\left.L^{2}\left([0, T], \mathbb{R}^{2}\right)\right)$

(3) For all $t \in[0, T]$ the map $x \rightarrow(Y(\cdot, t, x), Z(\cdot, t, x))$ is Gâteaux differentiable as a map from $H$ to $L_{\mathcal{P}}^{p}(\Omega, C([0, T], \mathbb{R})) \times L_{\mathcal{P}}^{p}\left(\Omega, L^{2}\left([0, T], \mathbb{R}^{2}\right)\right) ;$ moreover the map $(t, x, h) \rightarrow\left(\nabla_{x} Y(\cdot, t, x) h, \nabla_{x} Z(\cdot, t, x) h\right)$ is continuous from $[0, T] \times H \times H$ to $L_{\mathcal{P}}^{p}(\Omega, C([0, T], \mathbb{R})) \times L_{\mathcal{P}}^{p}\left(\Omega, L^{2}\left([0, T], \mathbb{R}^{2}\right)\right)$.

(4) The following equation holds for all $t \in[0, T], x, h \in H$.

$$
\left\{\begin{aligned}
& \mathrm{d} \nabla_{x} Y(s, t, x) h=\nabla_{x} \Psi(s, X(s, t, x), Z(s, t, x)) \nabla_{x} X(s, t, x) h \mathrm{~d} s \\
&+\nabla_{z} \Psi(s, X(s, t, x), Z(s, t, x)) \nabla_{x} Z(s, t, x) h \mathrm{~d} s+\nabla_{x} Z(s, t, x) h \mathrm{~d} W_{s}, \\
& \nabla_{x} Y(T, t, x) h=\nabla_{x} \Phi(X(T, t, x)) \nabla_{x} X(T, t, x) h, \quad s \in[t, T] .
\end{aligned}\right.
$$

Proof. The claim follows directly from Proposition 4.8 in [14], from Proposition 3.1 above and from the chain rule (in the form stated in Lem. 2.1 of [14]). 
Remark 4.2. The inequality (3.8), together with the inequality (4.9) in [14], implies that there exists a constant $C_{Y, p}$ such that for all $t \in[0, T]$ and $x \in H$

$$
\mathbb{E} \sup _{s \in[0, T]}|Y(s, t, x)|^{p}+\mathbb{E}\left(\int_{0}^{T}|Z(s, t, x)|^{2} \mathrm{~d} s\right)^{p / 2} \leq C_{Y, p}(1+|x|)^{2 p} .
$$

Remark 4.3. $Y(t, t, x)$ is adapted both to the $\sigma$-field $\sigma\left\{W_{s}: s \in[0, t]\right\}$ and to the $\sigma$-field $\sigma\left\{W_{s}-W_{t}: s \in[t, T]\right\}$. Thus $Y(t, t, x)$ and $\nabla Y(t, t, x) h, x, h \in H$ are deterministic.

Proposition 3.2 yields the following further regularity result.

Proposition 4.4. For every $\alpha \in[0,1 / 2), p \in[2, \infty)$ there exist two families of processes

$$
\left\{P^{\alpha}(s, t, x) k: s \in[0, T]\right\} \text { and }\left\{Q^{\alpha}(s, t, x) k: s \in[0, T]\right\} ; \quad t \in[0, T), x \in H, k \in H
$$

with $P^{\alpha}(\cdot, t, x) k \in L_{\mathcal{P}}^{p}(\Omega, C([0, T], \mathbb{R}))$ and $\left.Q^{\alpha}(\cdot, t, x) k\right) \in L_{\mathcal{P}}^{p}\left(\Omega, L^{2}\left([0, T], \mathbb{R}^{2}\right)\right)$ such that if $k \in D(-A)^{\alpha}, t \in$ $[0, T), x \in H$, then $\mathbb{P}$-a.s.

$$
\begin{gathered}
P^{\alpha}(s, t, x) k= \begin{cases}\nabla_{x} Y(s, t, x)(\lambda-A)^{\alpha} k & \text { for all } s \in[t, T], \\
\nabla_{x} Y(t, t, x)(\lambda-A)^{\alpha} k & \text { for all } s \in[0, t),\end{cases} \\
Q^{\alpha}(s, t, x) k= \begin{cases}\nabla_{x} Z(s, t, x)(\lambda-A)^{\alpha} k & \text { for a.e. } s \in[t, T], \\
0 & \text { if } s \in[0, t) .\end{cases}
\end{gathered}
$$

Moreover the map $(t, x, k) \rightarrow P^{\alpha}(\cdot, t, x) k$ is continuous from $[0, T) \times H \times H$ to $L_{\mathcal{P}}^{p}(\Omega, C([0, T], \mathbb{R}))$ and the map $(t, x, k) \rightarrow Q^{\alpha}(\cdot, t, x) k$ is continuous from $[0, T) \times H \times H$ to $L_{\mathcal{P}}^{p}\left(\Omega, L^{2}\left([0, T], \mathbb{R}^{2}\right)\right)$ and both maps are linear with respect to $k$.

Finally there exists a constant $C_{\nabla Y, \alpha, p}$ such that

$$
\mathbb{E} \sup _{s \in[0, T]}\left|P^{\alpha}(s, t, x) k\right|_{H}^{p}+\mathbb{E}\left(\int_{0}^{T}\left|Q^{\alpha}(s, t, x) k\right|_{\left(\mathbb{R}^{2}\right)^{*}}^{2} \mathrm{~d} s\right)^{p / 2} \leq C_{\nabla Y, \alpha, p}(T-t)^{-\alpha p}\left(1+|x|_{H}\right)^{p}|k|_{H}^{p} .
$$

Proof. Let, for $t \in[0, T], x \in H, k \in D(-A)^{\alpha}, P^{\alpha}(\cdot, t, x) k$ and $Q^{\alpha}(\cdot, t, x) k$ be defined by (4.4) and (4.5) respectively.

By Proposition 4.1 the map $k \rightarrow\left(P^{\alpha}(\cdot, t, x) k, Q^{\alpha}(\cdot, t, x) k\right)$ is a bounded linear operator from $D(-A)^{\alpha}$ to $L_{\mathcal{P}}^{p}(\Omega, C([0, T], \mathbb{R})) \times L_{\mathcal{P}}^{p}\left(\Omega, L^{2}\left([0, T], \mathbb{R}^{2}\right)\right)$. Moreover $\left(P^{\alpha}(\cdot, t, x) k, Q^{\alpha}(\cdot, t, x) k\right.$ solves the equation

$$
\left\{\begin{aligned}
\mathrm{d} P^{\alpha}(s, t, x) k & =1_{[t, T]}(s) \nabla_{x} \Psi(s, X(s, t, x), Z(s, t, x)) \nabla_{x} X(s, t, x)(\lambda-A)^{\alpha} k \mathrm{~d} s \\
+ & \nabla_{z} \Psi(s, X(s, t, x), Z(s, t, x)) Q^{\alpha}(s, t, x) k \mathrm{~d} s+Q^{\alpha}(s, t, x) k \mathrm{~d} W_{s}, \\
P^{\alpha}(T, t, x) k & =\nabla_{x} \Phi(X(T, t, x)) \nabla_{x} X(T, t, x)(\lambda-A)^{\alpha} k, \quad s \in[t, T] .
\end{aligned}\right.
$$

By (3.4) equation (4.7) can be rewritten

$$
\left\{\begin{array}{l}
\mathrm{d} P^{\alpha}(s, t, x) k=\nu(s, t, x) k \mathrm{~d} s+\nabla_{z} \Psi(s, X(s, t, x), Z(s, t, x)) Q^{\alpha}(s, t, x) k \mathrm{~d} s+Q^{\alpha}(s, t, x) k \mathrm{~d} W_{s} \\
P^{\alpha}(T, t, x) k=\eta(t, x) k, \quad s \in[0, T]
\end{array}\right.
$$

where

$$
\begin{aligned}
& \nu(s, t, x) k=1_{[t, T]}(s) \nabla_{x} \Psi(s, X(s, t, x), Z(s, t, x))\left(\Theta^{\alpha}(s, t, x) k+(\lambda-A)^{\alpha} \mathrm{e}^{(s-t) A} k\right) \\
& \eta(t, x) k=\nabla_{x} \Phi(X(T, t, x))\left(\Theta^{\alpha}(T, t, x) k+(\lambda-A)^{\alpha} \mathrm{e}^{(T-t) A} k\right) .
\end{aligned}
$$


Now we choose arbitrary $k \in H$ and notice that $\nu(s, t, x) k$ and $\eta(t, x) k$ can still be defined by the above formulae. Remark 2.7, and relations (2.4), (3.5) yield:

$$
\begin{aligned}
\mathbb{E}\left(\int_{0}^{T}|\nu(s, t, x) k|^{2} \mathrm{~d} s\right)^{p / 2} & \leq c_{1} \mathbb{E}\left(\int_{t}^{T}(1+|X(s, t, x)|)^{2}\left(\left|\Theta^{\alpha}(s, t, x) k\right|+(s-t)^{-\alpha}|k|_{H}\right)^{2} \mathrm{~d} s\right)^{p / 2} \\
& \leq c_{2}\left[(T-t)^{p / 2}+(T-t)^{(1-2 \alpha) p / 2}\right](1+|x|)^{p}|k|^{p} \leq c_{3}(1+|x|)^{p}|k|^{p}
\end{aligned}
$$

where $c_{1}, c_{2}$ and $c_{3}$ are suitable constants independent on $t, x, k$. In the same way

$$
\begin{aligned}
\mathbb{E}|\eta(t, x) k|^{p} & \leq c_{4} \mathbb{E}\left((1+|X(T, t, x)|)\left(\left|\Theta^{\alpha}(T, t, x) k\right|+(T-t)^{-\alpha}|k|_{H}\right)\right)^{p} \\
& \leq c_{5}(T-t)^{-p \alpha}(1+|x|)^{p}|k|^{p}
\end{aligned}
$$

By Proposition 4.3 in [14], for all $k \in H$ there exists a unique pair $\left(\left(P^{\alpha}(\cdot, t, x) k, Q^{\alpha}(\cdot, t, x) k\right)\right.$ belonging to $L_{\mathcal{P}}^{p}(\Omega, C([0, T], \mathbb{R})) \times L_{\mathcal{P}}^{p}\left(\Omega, L^{2}\left([0, T], \mathbb{R}^{2}\right)\right)$ and solving equation (4.8); moreover (4.6) holds. The map $k \rightarrow$ $\left(P^{\alpha}(\cdot, t, x) k, Q^{\alpha}(\cdot, t, x) k\right)$ is clearly linear, so we can conclude that the required extension exists. To prove its continuity, by the estimate (4.6) it is sufficient to show its continuity with respect to $t$ and $x$ for any fixed $k \in H$. Again by Proposition 4.3 in [14], it is enough to prove that for all $k \in H, t_{n} \rightarrow t \in[0, T), x_{n} \rightarrow x \in H$ and all $\mu \in L_{\mathcal{P}}^{p}\left(\Omega, L^{2}\left([0, T], \mathbb{R}^{2}\right)\right)$ letting

$$
\begin{gathered}
I_{1}:=\mathbb{E}\left(\int_{0}^{T}\left|\left(\nabla_{z} \Psi\left(s, X_{s}^{n}, Z_{s}^{n}\right)-\nabla_{z} \Psi\left(s, X_{s}, Z_{s}\right)\right) \mu_{s}\right|^{2} \mathrm{~d} s\right)^{p / 2}, \\
I_{2}:=\mathbb{E}\left(\int_{0}^{T}\left|\nu\left(s, t_{n}, x_{n}\right) h-\nu(s, t, x) h\right|^{2} \mathrm{~d} s\right)^{p / 2}, \\
I_{3}:=\mathbb{E}\left|\eta\left(t_{n}, x_{n}\right) k-\eta(t, x) k\right|^{p}
\end{gathered}
$$

where $X_{s}^{n}=X\left(s, t_{n}, x_{n}\right), Z_{s}^{n}=Z\left(s, t_{n}, x_{n}\right)$, then $I_{i} \rightarrow 0$, as $n \rightarrow \infty, i=1,2,3$. Notice that, for all $p \geq 1$, $X_{s}^{n} \rightarrow X(\cdot, t, x)$ in $L_{\mathcal{P}}^{p}(\Omega, C([0, T], H))$ and $Z_{s}^{n} \rightarrow Z(\cdot, t, x)$ in $L_{\mathcal{P}}^{p}\left(\Omega, L^{2}\left([0, T], \mathbb{R}^{2}\right)\right)$. Thus from each subsequence in $\mathbb{N}$ we can extract a subsequence $\left\{n_{i}: i \in \mathbb{N}\right\}$ for which $\sum_{i}\left|X^{n_{i}}-X(\cdot, t, x)\right|_{L_{\mathcal{P}}^{p}(\Omega, C([0, T], H))}<\infty$, and therefore the series $\sum_{i}\left(X^{n_{i}}-X(\cdot, t, x)\right)$ converges, $\mathbb{P}$-a.s. for all $s \in[0, T]$, to an element $X^{*}$ of $L_{\mathcal{P}}^{p}(\Omega, C([0, T], H))$ for which, $\mathbb{P}$-a.s., $\left|X_{s}^{n_{i}}\right|_{H} \leq|X(s, t, x)|_{H}+\left|X_{s}^{*}\right|_{H}$ for $s \in[0, T]$. Similarly, there exists $Z^{*} \in L_{\mathcal{P}}^{p}\left(\Omega, L^{2}\left([0, T], \mathbb{R}^{2}\right)\right)$ such that $\left|Z_{s}^{n_{i}}\right|_{\mathbb{R}^{2}} \leq|Z(s, t, x)|_{\mathbb{R}^{2}}+\left|Z_{s}^{*}\right|_{\mathbb{R}^{2}}, \mathbb{P}$-a.s. for almost all $s \in[0, T]$.

To prove (4.9) it is enough to notice that from each subsequence in $\mathbb{N}$ we can extract a subsequence $\left\{n_{i}: i \in \mathbb{N}\right\}$ as described above and by the dominated convergence theorem we have

$$
E\left(\int_{0}^{T}\left|\left(\nabla_{z} \Psi\left(s, X_{s}^{n_{i}}, Z_{s}^{n_{i}}\right)-\nabla_{z} \Psi\left(s, X_{s}, Z_{s}\right)\right) \mu_{s}\right|^{2} \mathrm{~d} s\right)^{p / 2} \rightarrow 0
$$

To prove (4.10) we define:

$$
\begin{gathered}
V_{s}^{n}=1_{\left[t_{n}, T\right]}(s)\left(\Theta^{\alpha}\left(s, t_{n}, x_{n}\right) k+(\lambda-A)^{\alpha} \mathrm{e}^{\left(s-t_{n}\right) A} k\right), \\
V_{s}=1_{[t, T]}(s)\left(\Theta^{\alpha}(s, t, x) k+(\lambda-A)^{\alpha} \mathrm{e}^{(s-t) A} k\right),
\end{gathered}
$$


and notice that $\nu\left(s, t_{n}, x_{n}\right)=\nabla_{x} \Psi\left(s, X_{s}^{n}, Z_{s}^{n}\right) V_{s}^{n}$ and $\nu(s, t, x)=\nabla_{x} \Psi\left(s, X_{s}, Z_{s}\right) V_{s}$. Then

$$
\begin{aligned}
I_{2} \leq & 2^{p-1} \mathbb{E}\left(\int_{0}^{T}\left|\left(\nabla_{x} \Psi\left(s, X_{s}^{n}, Z_{s}^{n}\right)-\nabla_{x} \Psi\left(s, X_{s}, Z_{s}\right)\right) V_{s}\right|^{2} \mathrm{~d} s\right)^{p / 2} \\
& +2^{p-1} \mathbb{E}\left(\int_{0}^{T}\left|\nabla_{x} \Psi\left(s, X_{s}^{n}, Z_{s}^{n}\right)\left(V_{s}^{n}-V_{s}\right)\right|^{2} \mathrm{~d} s\right)^{p / 2} \\
= & I_{21}+I_{22} .
\end{aligned}
$$

Taking into account the inequalities $\left|\nabla_{x} \Psi(s, x, z)\right| \leq c(1+|x|)$ and $\left|V_{s}\right| \leq c 1_{[t, T]}(s)\left(1+(t-s)^{-\alpha}\right)$, and applying the estimate (2.4) (with $\alpha=0$ ) to $X$ and $X^{n}, I_{21}$ can be shown to tend to zero as it was done for $I_{1}$. To show that $I_{22} \rightarrow 0$, by the Hölder inequality it is sufficient to show that $\nabla_{x} \Psi\left(\cdot, X^{n}, Z^{n}\right)$ is bounded in $L_{\mathcal{P}}^{p}\left(\Omega, L^{r}\left([0, T], H^{*}\right)\right)$ for every $p, r \in[2, \infty)$ and that $V^{n} \rightarrow V$ in $L_{\mathcal{P}}^{p}\left(\Omega, L^{q}([0, T], H)\right)$ for every $p \in[2, \infty)$ and for some $q>2$. The required boundedness of $\nabla_{x} \Psi\left(\cdot, X^{n}, Z^{n}\right)$ follows immediately from the inequality $\left|\nabla_{x} \Psi(s, x, z)\right| \leq c(1+|x|)$ and the estimate (2.4) (with $\alpha=0$ ). To prove that $V^{n} \rightarrow V$ in the required norm we first note that

$$
\mathbb{E}\left(\int_{0}^{T}\left|1_{\left[t_{n}, T\right]}(s) \Theta^{\alpha}\left(s, t_{n}, x_{n}\right) k-1_{[t, T]}(s) \Theta^{\alpha}(s, t, x) k\right|^{q} \mathrm{~d} s\right)^{p / q} \rightarrow 0
$$

since the map $(t, x) \rightarrow \Theta^{\alpha}(\cdot, t, x) k$ is continuous with values in $L_{\mathcal{P}}^{p}(\Omega, C([0, T], H))$. So to conclude the proof that $I_{22} \rightarrow 0$ it remains to show that

$$
1_{\left[t_{n}, T\right]}(\cdot)(\lambda-A)^{\alpha} \mathrm{e}^{\left(\cdot-t_{n}\right) A} k \rightarrow 1_{[t, T]}(\cdot)(\lambda-A)^{\alpha} \mathrm{e}^{(\cdot-t) A} k
$$

in $L^{q}([0, T] ; H)$ for some $q>2$. To this end we note that for all $t_{n}^{+} \searrow t, t_{n}^{-} \nearrow t, x_{n}^{+} \rightarrow x, x_{n}^{-} \rightarrow x$ we have

$$
\begin{aligned}
& \int_{0}^{T}\left|(\lambda-A)^{\alpha}\left(1_{\left[t_{n}^{-}, T\right]}(s) \mathrm{e}^{\left(s-t_{n}^{-}\right) A}-1_{\left[t_{n}^{+}, T\right]}(s) \mathrm{e}^{\left(s-t_{n}^{+}\right) A}\right) k\right|^{q} \mathrm{~d} s \\
& =\int_{t_{n}^{-}}^{t_{n}^{+}}\left|(\lambda-A)^{\alpha} \mathrm{e}^{\left(s-t_{n}^{-}\right) A} k\right|^{q} \mathrm{~d} s+\int_{t_{n}^{+}}^{T}\left|(\lambda-A)^{\alpha} \mathrm{e}^{\left(s-t_{n}^{+}\right) A}\left(\mathrm{e}^{\left(t_{n}^{+}-t_{n}^{-}\right) A}-I\right) k\right|^{q} \mathrm{~d} s \\
& \quad \leq|k|^{q} \int_{t_{n}^{-}}^{t_{n}^{+}}\left(s-t_{n}^{-}\right)^{-q \alpha} \mathrm{d} s+\left|\left(\mathrm{e}^{\left(t_{n}^{+}-t_{n}^{-}\right) A}-I\right) k\right|^{q} \int_{t_{n}^{+}}^{T}\left(s-t_{n}^{+}\right)^{-q \alpha} \mathrm{d} s \rightarrow 0,
\end{aligned}
$$

provided $q$ is sufficiently close to 2 , since $\alpha<1 / 2$.

Finally, the proof of (4.11) is similar to the proof of (4.10).

Corollary 4.5. Setting $v(t, x)=Y(t, t, x)$, we have $v \in C([0, T] \times H ; \mathbb{R})$ and there exists a constant $C$ such that $|v(t, x)| \leq C(1+|x|)^{2}, t \in[0, T], x \in H$. Moreover $v$ is Gâteaux differentiable with respect to $x$ on $[0, T] \times H$ and the map $(t, x, h) \rightarrow \nabla v(t, x) h$ is continuous.

For all $\alpha \in[0,1 / 2), t \in[0, T)$ and $x \in H$ the linear operator $k \rightarrow \nabla v(t, x)(\lambda-A)^{\alpha} k$-a priori defined for $k \in D(-A)^{\alpha}$ - has an extension to a bounded linear operator $H \rightarrow \mathbb{R}$, that we denote by $\left[\nabla v(\lambda-A)^{\alpha}\right](t, x)$.

Finally the map $(t, x, k) \rightarrow\left[\nabla v(\lambda-A)^{\alpha}\right](t, x) k$ is continuous $[0, T) \times H \times H \rightarrow \mathbb{R}$ and there exists $C_{\nabla v, \alpha}$ for which:

$$
\left|\left[\nabla v(\lambda-A)^{\alpha}\right](t, x) k\right| \leq C_{\nabla v, \alpha}(T-t)^{-\alpha}\left(1+|x|_{H}\right)|k|_{H}, \quad t \in[0, T), x, k \in H .
$$

Proof. We recall that $Y(t, t, x)$ is deterministic.

Since the map $(t, x) \rightarrow Y(\cdot, t, x)$ is continuous with values in $L_{\mathcal{P}}^{p}(\Omega, C([0, T], \mathbb{R})), p \geq 2$, then the map $(t, x) \rightarrow Y(t, t, x)$ is continuous with values in $L^{p}(\Omega, \mathbb{R})$ and so the map $(t, x) \rightarrow \mathbb{E} Y(t, t, x)=Y(t, t, x)=v(t, x)$ is continuous with values in $\mathbb{R}$. 
Similarly, $\nabla_{x} v(t, x)=\mathbb{E} \nabla_{x} Y(t, t, x)$ exists and has the required continuity properties, by Proposition 4.1 .

Next we notice that $P^{\alpha}(t, t, x) k=\nabla_{x} Y(t, t, x)(\lambda-A)^{\alpha} k$. The existence of the required extensions and its continuity are direct consequences of Proposition 4.4. Finally the estimate (4.12) follows from (4.6).

Remark 4.6. It is evident by construction that the law of $Y(\cdot, t, x)$ and consequently the function $v$ depends on the law of the Wiener process $W$ but not on the particular probability $\mathbb{P}$ and Wiener process $W$ we have chosen.

Corollary 4.7. For every $t \in[0, T], x \in H$ we have, $\mathbb{P}$-a.s.,

$$
\begin{gathered}
Y(s, t, x)=v(s, X(s, t, x)), \quad \text { for all } s \in[t, T] \\
Z(s, t, x)=\left[\nabla v(\lambda-A)^{1-\beta}\right](s, X(s, t, x))(\lambda-A)^{\beta} b, \quad \text { for almost all } s \in[t, T] .
\end{gathered}
$$

Proof. We start from the well-known equality: for $0 \leq t \leq r \leq T$, $\mathbb{P}$-a.s.,

$$
X(s, t, x)=X(s, r, X(r, t, x)), \quad \text { for all } s \in[r, T] .
$$

It follows easily from the uniqueness of the backward equation (4.1) that $\mathbb{P}$-a.s.,

$$
Y(s, t, x)=Y(s, r, X(r, t, x)), \quad \text { for all } s \in[r, T]
$$

Setting $s=r$ we arrive at (4.13).

To prove (4.14) we note that it follows immediately from the backward equation (4.1) that the joint quadratic variation of $\{Y(s, t, x), s \in[t, T]\}$ and $W^{j}(j=1,2)$ on an arbitrary interval $[t, s] \subset[t, T)$ is equal to $\int_{t}^{s} Z_{r}^{j} \mathrm{~d} r$. By (4.13) the same result can be obtained by considering the joint quadratic variation of $\{v(s, X(s, t, x)), s \in$ $[t, T]\}$ and $W^{j}$. An application of Proposition 3.5 (whose assumptions hold true by Cor. 4.5) leads to the identity

and (4.14) is proved.

$$
\int_{t}^{s} Z_{r}^{j} \mathrm{~d} r=\int_{t}^{s}\left[\nabla v(\lambda-A)^{1-\beta}\right](r, X(r, t, x))(\lambda-A)^{\beta} b^{j} \mathrm{~d} r
$$

\section{The Hamilton-Jacobi-Bellman equation}

Let us consider again the solution $X(s, t, x)$ of equation $(2.2)$ and denote by $P_{t, s}$ its transition semigroup:

$$
P_{t, s}[\phi](x)=\mathbb{E} \phi(X(s, t, x)), \quad x \in H, 0 \leq t \leq s \leq T,
$$

for any bounded measurable $\phi: H \rightarrow \mathbb{R}$. We note that by the estimate (2.4) (with $\alpha=0$ ) this formula is meaningful for every $\phi$ with polynomial growth. In the following $P_{t, s}$ will be considered as an operator acting on this class of functions.

Let us denote by $\mathcal{L}_{t}$ the generator of $P_{t, s}$, formally:

$$
\mathcal{L}_{t}[\phi](x)=\frac{1}{2}\left\langle\nabla^{2} \phi(x)(\lambda-A) b^{1},(\lambda-A) b^{1}\right\rangle+\frac{1}{2}\left\langle\nabla^{2} \phi(x)(\lambda-A) b^{2},(\lambda-A) b^{2}\right\rangle+\langle A x+F(t, x), \nabla \phi(x)\rangle,
$$

where $\nabla \phi(x)$ and $\nabla^{2} \phi(x)$ are first and second Gâteaux derivatives of $\phi$ at the point $x \in H$ (here they are identified with elements of $H$ and $L(H)$ respectively).

The Hamilton-Jacobi-Bellman equation for the optimal control problem is

$$
\left\{\begin{array}{l}
\frac{\partial v(t, x)}{\partial t}+\mathcal{L}_{t}[v(t, \cdot)](x)=\Psi(t, x, \nabla v(t, x)(\lambda-A) b), \quad t \in[0, T], x \in H \\
v(T, x)=\Phi(x)
\end{array}\right.
$$


This is a nonlinear parabolic equation for the unknown function $v:[0, T] \times H \rightarrow \mathbb{R}$. The operators $\mathcal{L}_{t}$ are very degenerate, since the space $H$ is infinite-dimensional but the noise $W$ is a two-dimensional Wiener process.

Now we consider the variation of constants formula for (5.1):

$$
v(t, x)=P_{t, T}[\Phi](x)-\int_{t}^{T} P_{t, s}[\Psi(s, \cdot, \nabla v(s, \cdot)(\lambda-A) b)](x) \mathrm{d} s, \quad t \in[0, T], x \in H .
$$

This equality is still formal, since the term $(\lambda-A) b$ is not defined. However with a slightly different interpretation we arrive at the following precise definition:

Definition 5.1. We say that a function $v:[0, T] \times H \rightarrow \mathbb{R}$ is a mild solution of the Hamilton-Jacobi-Bellman equation (5.1) if the following conditions hold:

(i) $v \in C([0, T] \times H ; \mathbb{R})$ and there exist constants $C, m \geq 0$ such that $|v(t, x)| \leq C(1+|x|)^{m}, t \in[0, T]$, $x \in H$.

(ii) $v$ is Gâteaux differentiable with respect to $x$ on $[0, T) \times H$ and the map $(t, x, h) \rightarrow \nabla v(t, x) h$ is continuous $[0, T) \times H \times H \rightarrow \mathbb{R}$.

(iii) For all $t \in[0, T)$ and $x \in H$ the linear operator $k \rightarrow \nabla v(t, x)(\lambda-A)^{1-\beta} k$ (a priori defined for $\left.k \in D(-A)^{1-\beta}\right)$ has an extension to a bounded linear operator $H \rightarrow \mathbb{R}$, that we denote by $[\nabla v(\lambda-$ $\left.A)^{1-\beta}\right](t, x)$.

Moreover the map $(t, x, k) \rightarrow\left[\nabla v(\lambda-A)^{1-\beta}\right](t, x) k$ is continuous $[0, T) \times H \times H \rightarrow \mathbb{R}$ and there exist constants $C, m \geq 0, \kappa \in[0,1)$ such that

$$
\left|\left[\nabla v(\lambda-A)^{1-\beta}\right](t, x)\right|_{H^{*}} \leq C(T-t)^{-\kappa}(1+|x|)^{m}, \quad t \in[0, T), x \in H .
$$

(iv) The following equality holds for every $t \in[0, T], x \in H$ :

$$
v(t, x)=P_{t, T}[\Phi](x)-\int_{t}^{T} P_{t, s}\left[\Psi\left(s, \cdot,\left[\nabla v(\lambda-A)^{1-\beta}\right](s, \cdot)(\lambda-A)^{\beta} b\right)\right](x) \mathrm{d} s .
$$

We note that Proposition 2.5 implies that $|\Psi(s, x, z)| \leq C\left(1+|z|+|x|^{2}\right)$ and it follows that

$$
\left|\Psi\left(s, x,\left[\nabla v(\lambda-A)^{1-\beta}\right](s, x)(\lambda-A)^{\beta} b\right)\right| \leq C(T-t)^{-\kappa}(1+|x|)^{m+2},
$$

and using the estimate (2.4) (with $\alpha=0$ ) it is easy to conclude that formula (5.3) is meaningful.

Theorem 5.1. Assume Hypotheses 2.1, 2.2 and 2.6 then there exists a unique mild solution of the HamiltonJacobi-Bellman equation (5.1).

The solution $v$ is given by the formula

$$
v(t, x)=Y(t, t, x),
$$

where $(X, Y, Z)$ is the solution of the forward-backward system (2.3)-(4.2).

Proof. Existence. By Corollary 4.5 the solution $v$ has the regularity properties stated in Definition 5.1. In order to verify that equality (5.3) holds we first fix $t \in[0, T]$ and $x \in H$ and note that the backward equation (4.1) gives

$$
Y(t, t, x)+\int_{t}^{T} Z_{s} \mathrm{~d} W_{s}=\Phi(X(T, t, x))-\int_{t}^{T} \Psi(s, X(s, t, x), Z(s, t, x)) \mathrm{d} s .
$$

Taking expectation we obtain

$$
v(t, x)=P_{t, T}[\Phi](x)-\mathbb{E} \int_{t}^{T} \Psi(s, X(s, t, x), Z(s, t, x)) \mathrm{d} s .
$$


Now we recall that by Corollary 4.7 we have

$$
Z(s, t, x)=\left[\nabla v(\lambda-A)^{1-\beta}\right](s, X(s, t, x))(\lambda-A)^{\beta} b, \quad \mathbb{P} \text {-a.s. for a.a. } s \in[t, T] .
$$

It follows that

$$
\begin{aligned}
\mathbb{E} \int_{t}^{T} \Psi(s, X(s, t, x), Z(s, t, x)) \mathrm{d} s & =\mathbb{E} \int_{t}^{T} \Psi\left(s, X(s, t, x),\left[\nabla v(\lambda-A)^{1-\beta}\right](s, X(s, t, x))(\lambda-A)^{\beta} b\right) \mathrm{d} s \\
& =\int_{t}^{T} P_{t, s}\left[\Psi\left(s, \cdot\left[\nabla v(\lambda-A)^{1-\beta}\right](s, \cdot)(\lambda-A)^{\beta} b\right)\right](x) \mathrm{d} s .
\end{aligned}
$$

Comparing with (5.4) gives the required equality (5.3).

Uniqueness. Let $u$ be a mild solution. We fix $t \in[0, T]$ and $x \in H$ and look for a convenient expression for the process $u(s, X(s, t, x)), s \in[t, T]$. By (5.3) and the Markov property of $X$ we have

$$
\begin{aligned}
u(s, X(s, t, x)) & =P_{s, T}[\Phi](X(s, t, x))-\int_{s}^{T} P_{s, r}\left[\Psi\left(r, \cdot,\left[\nabla u(\lambda-A)^{1-\beta}\right](r, \cdot)(\lambda-A)^{\beta} b\right)\right](X(s, t, x)) \mathrm{d} r \\
& =\mathbb{E}^{\mathcal{F}_{s}}[\Phi(X(T, t, x))]-\mathbb{E}^{\mathcal{F}_{s}}\left[\int_{s}^{T} \Psi\left(r, X(r, t, x),\left[\nabla u(\lambda-A)^{1-\beta}\right](r, X(r, t, x))(\lambda-A)^{\beta} b\right) \mathrm{d} r\right] \\
& =\mathbb{E}^{\mathcal{F}_{s}}[\xi]+\int_{t}^{s} \Psi\left(r, X(r, t, x),\left[\nabla u(\lambda-A)^{1-\beta}\right](r, X(r, t, x))(\lambda-A)^{\beta} b\right) \mathrm{d} r
\end{aligned}
$$

where we have defined

$$
\xi=\Phi(X(T, t, x))-\int_{t}^{T} \Psi\left(r, X(r, t, x),\left[\nabla u(\lambda-A)^{1-\beta}\right](r, X(r, t, x))(\lambda-A)^{\beta} b\right) \mathrm{d} r
$$

We note that $\mathbb{E}^{\mathcal{F}_{t}}[\xi]=\mathbb{E}^{\mathcal{F}_{t}} u(s, X(s, t, x))=u(t, x)$. Since $\xi \in L^{2}(\Omega ; \mathbb{R})$ is $\mathcal{F}_{T}$-measurable, by a well known representation theorem there exists $\widetilde{Z} \in L_{\mathcal{P}}^{2}\left(\Omega \times[t, T] ; \mathbb{R}^{2}\right)$ such that $\mathbb{E}^{\mathcal{F}_{s}}[\xi]=\int_{t}^{s} \widetilde{Z}_{r} \mathrm{~d} W_{r}+u(t, x)$. We conclude that the process $u(s, X(s, t, x)), s \in[t, T]$ is a (real) continuous semimartingale with canonical decomposition

$$
u(s, X(s, t, x))=u(t, x)+\int_{t}^{s} \widetilde{Z}_{r} \mathrm{~d} W_{r}+\int_{t}^{s} \Psi\left(r, X(r, t, x),\left[\nabla u(\lambda-A)^{1-\beta}\right](r, X(r, t, x))(\lambda-A)^{\beta} b\right) \mathrm{d} r
$$

into its continuous martingale part and its continuous finite variation part.

Now we compute the joint quadratic variation process of both sides of $(5.5)$ with $W^{j}, j=1,2$, on an arbitrary interval $[0, s] \subset[0, T)$. By (5.2) there exists a constant $K_{s}$ such that $|\nabla u(t, x)| \leq K_{s}(1+|x|)^{m}$, for $t \in[0, s]$, $x \in H$; then we can apply Proposition 3.5 and conclude that the joint quadratic variation equals

$$
\int_{0}^{s}\left[\nabla u(\lambda-A)^{1-\beta}\right](r, X(r, t, x))(\lambda-A)^{\beta} b^{j} \mathrm{~d} r
$$

Computing the joint quadratic variation of the left-hand side of (5.5) with $W^{j}$ yields the identity

$$
\int_{0}^{s}\left[\nabla u(\lambda-A)^{1-\beta}\right](r, X(r, t, x))(\lambda-A)^{\beta} b^{j} \mathrm{~d} r=\int_{t}^{s} \widetilde{Z}_{r}^{j} \mathrm{~d} r
$$


Therefore, for a.a. $s \in[t, T]$, we have $\mathbb{P}$-a.s., $\left[\nabla u(\lambda-A)^{1-\beta}\right](s, X(s, t, x))(\lambda-A)^{\beta} b=\widetilde{Z}_{s}$, so substituting into (5.5) and taking into account that $u(T, X(T, t, x))=\Phi(X(T, t, x))$ we obtain, for $s \in[t, T]$,

$$
\begin{gathered}
u(s, X(s, t, x))+\int_{s}^{T}\left[\nabla u(\lambda-A)^{1-\beta}\right](r, X(r, t, x))(\lambda-A)^{\beta} b \mathrm{~d} W_{r} \\
=\Phi(X(T, t, x))-\int_{s}^{T} \Psi\left(r, X(r, t, x),\left[\nabla u(\lambda-A)^{1-\beta}\right](r, X(r, t, x))(\lambda-A)^{\beta} b\right) \mathrm{d} r .
\end{gathered}
$$

Comparing with the backward equation (4.1) we note that the pairs

$$
(Y(s, t, x), Z(s, t, x)) \text { and }\left(u(s, X(s, t, x)),\left[\nabla u(\lambda-A)^{1-\beta}\right](s, X(s, t, x))(\lambda-A)^{\beta} b^{j}\right), s \in[t, T]
$$

solve the same equation. By uniqueness, we have $Y(s, t, x)=u(s, X(s, t, x)), s \in[t, T]$, and setting $s=t$ we obtain $Y(t, t, x)=u(t, x)$.

\section{Synthesis OF THE OPTIMAL CONTROL}

\subsection{Strong formulation}

In this section we assume that Hypotheses 2.1, 2.2 and 2.6 are satisfied and we come back to the control problem introduced in Section 2. As in the previous sections, $W$ is a standard real Wiener processes in $\mathbb{R}^{2}$, defined on a probability space $(\Omega, \mathcal{F}, \mathbb{P})$, with respect to a filtration $\left\{\mathcal{F}_{t}, t \geq 0\right\}$ satisfying the usual conditions. We call $u=\left(u^{1}, u^{2}\right): \Omega \times[0, T] \rightarrow \mathbb{R}^{2}$ an admissible control process if it is $\left(\mathcal{F}_{t}\right)$-predictable and takes values in the set $\mathcal{U} \subset \mathbb{R}^{2}$.

For arbitrary $t \in[0, T]$ and $x \in H$ we wish to minimize the cost $(2.6)$ :

$$
J(t, x, u(\cdot))=\mathbb{E} \int_{t}^{T} L\left(s, X_{s}^{u}, u(s)\right) \mathrm{d} s+\mathbb{E} \Phi\left(X_{T}^{u}\right)
$$

over all admissible controls, where $\left\{X_{s}^{u}: s \in[t, T]\right\}$ is the solution of the state equation (2.2): $\mathbb{P}$-a.s.,

$$
\begin{aligned}
X_{s}^{u}=\mathrm{e}^{(s-t) A} x+\int_{t}^{s} \mathrm{e}^{(s-r) A} F\left(r, X_{r}^{u}\right) \mathrm{d} r+\int_{t}^{s}(\lambda-A)^{1-\beta} \mathrm{e}^{(s-r) A}(\lambda-A)^{\beta} b \mathrm{~d} W_{r} & \\
& +\int_{t}^{s}(\lambda-A)^{1-\beta} \mathrm{e}^{(s-r) A}(\lambda-A)^{\beta} b u(r) \mathrm{d} r, \quad s \in[t, T] .
\end{aligned}
$$

We will also write $X^{u}(s, t, x)$ instead of $X_{s}^{u}$, to stress dependence on the initial data $t, x$.

We also recall, see Section 2.3 , that we denote by $\Gamma$ the set-valued mapping introduced in $(2.7)$ and by $\gamma:[0, T] \times H \times \mathbb{R}^{2} \rightarrow \mathcal{U}$ a measurable selection of $\Gamma$.

Finally, by $v:[0, T] \times H \rightarrow \mathbb{R}$, we denote the mild solution of the Hamilton-Jacobi-Bellman equation (5.1).

Theorem 6.1. Assume Hypotheses 2.1, 2.2 and 2.6. For every $t \in[0, T], x \in H$ and for all admissible control $u$ we have $J(t, x, u(\cdot)) \geq v(t, x)$, and the equality $J(t, x, u(\cdot))=v(t, x)$ holds if and only if

$$
u(s) \in \Gamma\left(s, X^{u}(s, t, x),\left[\nabla v(\lambda-A)^{1-\beta}\right]\left(s, X^{u}(s, t, x)\right)(\lambda-A)^{\beta} b\right)
$$

Proof. The proof is identical to the proof of relation (7.5) in [14], Theorem 7.2. Just notice that in this case by (4.14) we have $Z(s, t, x)=\left[\nabla v(\lambda-A)^{1-\beta}\right](s, X(s, t, x))(\lambda-A)^{\beta} b$ and the role of $G$ in [14], Theorem 7.2, is here played by $(\lambda-A) b$. 
The following corollary is now an immediate consequence. We nevertheless notice in the claim below we do not state conditions for the existence of a solution of the closed loop equation. Indeed existence is not obvious, due to the lack of regularity of the feedback law $\underline{u}$ occurring in (6.5). In the next section we will show that the closed loop equation admits a solution in a different, weak, sense. After reformulating the optimal control problem in the appropriate, weak, sense, this will lead to existence of the optimal control and the validity of the feedback law for an optimal pair.

However, under additional assumptions, it is also possible to solve the closed loop equation in the sense of (6.5) and therefore obtain existence of an optimal control in the present strong formulation. A slight modification of Proposition 2.4 shows that the Lipschitz condition

$$
\left|\underline{u}\left(t, x_{1}\right)-\underline{u}\left(t, x_{2}\right)\right| \leq C\left|x_{1}-x_{1}\right|, \quad t \in[0, T], x_{1}, x_{2} \in H
$$

for some constant $C$, is sufficient to ensure existence of the solution. In order to guarantee that (6.3) holds it is enough to require a uniform Lipschitz condition on the function $\gamma(t, \cdot, \cdot)$ and to prove that $\left[\nabla v(\lambda-A)^{1-\beta}\right](t, \cdot)$ is also Lipschitz. In turn, this could be deduced from properties of the backward equation (4.1), under additional assumptions on its coefficients $\Psi$ and $\Phi$, by the same approach as in Corollary 4.5 .

Corollary 6.2. Under the assumptions of Theorem 6.1, let us define

$$
\underline{u}(t, x)=\gamma\left(t, x,\left[\nabla v(\lambda-A)^{1-\beta}\right](t, x)(\lambda-A)^{\beta} b\right), \quad t \in[0, T], x \in H
$$

and let us assume that there exists an adapted process $\left\{\bar{X}_{s}, s \in[t, T]\right\}$ with continuous trajectories solving the equation: $\mathbb{P}$-a.s.

$$
\begin{aligned}
\bar{X}_{s}=\mathrm{e}^{(s-t) A} x_{0}+\int_{t}^{s} \mathrm{e}^{(s-r) A} F\left(r, \bar{X}_{r}\right) \mathrm{d} r & +\int_{t}^{s}(\lambda-A)^{1-\beta} \mathrm{e}^{(s-r) A}(\lambda-A)^{\beta} b \mathrm{~d} W_{r} \\
& +\int_{t}^{s}(\lambda-A)^{1-\beta} \mathrm{e}^{(s-r) A}(\lambda-A)^{\beta} b \underline{u}\left(r, \bar{X}_{r}\right) \mathrm{d} r, \quad s \in[t, T] .
\end{aligned}
$$

Then setting $\bar{u}(s)=\underline{u}\left(s, \bar{X}_{s}\right)$ we have $J(t, x, \bar{u}(\cdot))=v(t, x)$ and consequently the pair $(\bar{u}, \bar{X})$ is optimal for the control problem.

The function $\underline{u}$ introduced in (6.4) will be called the optimal feedback law, and the equation (6.5) is called the closed loop equation.

\subsection{Weak formulation}

We now reformulate the optimal control problem in the weak sense, following the approach of [13]. The main advantage is that we will be able to solve the closed loop equation, and hence to find an optimal control, although the feedback law $\underline{u}$ is non-smooth.

We still assume we are given the functions $f, \ell, \phi$ (and the corresponding functions $F, \Psi, \Phi$ ) and the set $\mathcal{U}$ as in the previous sections, satisfying Hypotheses 2.1, 2.2 and 2.6. We also assume that initial data $t \in[0, T]$ and $x \in H$ are given.

As in the previous section $\Gamma$ is the set-valued mapping introduced in $(2.7)$ and $\gamma:[0, T] \times H \times \mathbb{R}^{2} \rightarrow \mathcal{U}$ is an arbitrary measurable selection of $\Gamma$. Moreover $\underline{u}$ is defined as in (6.4).

We call $\left(\Omega, \mathcal{F},\left(\mathcal{F}_{t}\right), \mathbb{P}, W\right)$ an admissible set-up, or simply a set-up, if $(\Omega, \mathcal{F}, \mathbb{P})$ is a complete probability space with a right-continuous and $\mathbb{P}$-complete filtration $\left\{\mathcal{F}_{t}, t \in[0, T]\right\}$, and $\left\{W_{t}, t \in[0, T]\right\}$ is a standard $\mathbb{P}$-Wiener process with values in $\mathbb{R}^{2}$, with respect to the filtration $\left(\mathcal{F}_{t}\right)$.

An admissible control system (a.c.s.) is defined as $\mathbb{U}=\left(\Omega, \mathcal{F},\left(\mathcal{F}_{t}\right), \mathbb{P}, W, u, X^{u}\right)$ where:

- $\left(\Omega, \mathcal{F},\left(\mathcal{F}_{t}\right), \mathbb{P}, W\right)$ is an admissible set-up;

- $u: \Omega \times[0, T] \rightarrow \mathbb{R}^{2}$ is an $\left(\mathcal{F}_{t}\right)$-predictable process with values in $\mathcal{U}$; 
- $\left\{X_{s}^{u}, s \in[t, T]\right\}$ is an $\left(\mathcal{F}_{t}\right)$-adapted continuous process with values in $H$, mild solution of the state equation (6.2) with initial condition $X_{t}^{u}=x$.

By Proposition 2.4, on an arbitrary set-up the process $X^{u}$ is uniquely determined by $u$ and $x$, up to indistinguishability. To every a.c.s. we associate the cost $J(t, x, \mathbb{U})$ given by the right-hand side of (6.1). Although formally the same, it is important to note that now the cost is a functional of the a.c.s., and not a functional of $u$ alone. Our purpose is to minimize the functional $J(t, x, \mathbb{U})$ over all a.c.s. for fixed initial data $t, x$.

Our main result, Theorem 6.4 below, is based on the solvability of the closed loop equation (6.5) in the sense of the following lemma.

Lemma 6.3. For every $t \in[0, T], x \in H$, there exists an admissible set-up $\left(\Omega, \mathcal{F},\left(\mathcal{F}_{t}\right), \mathbb{P}, W\right)$ and an $\left(\mathcal{F}_{t}\right)$ adapted continuous process $\left\{\bar{X}_{s}, s \in[t, T]\right\}$ with values in $H$, solution of (6.5).

Proof. Let us take an arbitrary set-up $\left(\Omega, \mathcal{F},\left\{\mathcal{F}_{t}\right\}, \overline{\mathbb{P}}, \bar{W}\right)$ and consider the solution $\left\{\bar{X}_{s}: s \in[t, T]\right\}$ of the uncontrolled equation (compare with (2.3)):

$$
\bar{X}_{s}=\mathrm{e}^{(s-t) A} x+\int_{t}^{s} \mathrm{e}^{(s-r) A} F\left(r, \bar{X}_{r}\right) \mathrm{d} r+\int_{t}^{s}(\lambda-A)^{1-\beta} \mathrm{e}^{(s-r) A}(\lambda-A)^{\beta} b \mathrm{~d} \bar{W}_{r}, \quad s \in[t, T] .
$$

Now we define the process

$$
W_{s}=\bar{W}_{s}-\int_{t}^{s \vee t} \underline{u}\left(r, \bar{X}_{r}\right) \mathrm{d} r, \quad s \in[0, T] .
$$

Since $\underline{u}$ takes values in $\mathcal{U}$, it is bounded, and by the Girsanov Theorem there exists a probability $\mathbb{P}$ on $(\Omega, \mathcal{F})$, equivalent to $\overline{\mathbb{P}}$, such that $W$ is a $\mathbb{P}$-Wiener process with respect to $\left(\mathcal{F}_{t}\right)$. Re-writing equation (6.6) in terms of $W$ we conclude that $\bar{X}$ is the required solution of (6.5).

Using standard terminology we will say that $X$ is a weak solution of the closed loop equation (6.5).

Theorem 6.4. Assume Hypotheses $2.1,2.2$ and 2.6. For every $t \in[0, T], x \in H$, the infimum of $J(t, x, \mathbb{U})$ over all a.c.s. is equal to $v(t, x)$. Moreover there exists an a.c.s. $\mathbb{U}=\left(\Omega, \mathcal{F},\left(\mathcal{F}_{t}\right), \mathbb{P}, W, u, X^{u}\right)$ for which $J(t, x, \mathbb{U})=v(t, x)$ and the feedback law

$$
u(s)=\underline{u}\left(s, X_{s}^{u}\right), \quad \mathbb{P} \text {-a.s. for a.a. } s \in[t, T] .
$$

is verified by $u$ and $X^{u}$. Finally, the optimal trajectory $X^{u}$ is a weak solution of the closed loop equation.

Proof. Let $\left(\Omega, \mathcal{F},\left(\mathcal{F}_{t}\right), \mathbb{P}, W\right)$ and $\left\{\bar{X}_{s}, s \in[t, T]\right\}$ be the admissible set-up and the weak solution of the closedloop equation (6.5) given by Lemma 6.3. We can apply Theorem 6.1 and Corollary 6.2 with respect to this set-up and we obtain all the required conclusions.

\section{REFERENCES}

[1] P. Albano and P. Cannarsa, Lectures on carleman estimates for elliptic and parabolic operators with applications. Preprint, Università di Roma Tor Vergata.

[2] S. Albeverio and Y.A. Rozanov, On stochastic boundary conditions for stochastic evolution equations. Teor. Veroyatnost. $i$ Primenen. 38 (1993) 3-19.

[3] E. Alòs and S. Bonaccorsi, Stochastic partial differential equations with Dirichlet white-noise boundary conditions. Ann. Inst. H. Poincaré Probab. Statist. 38 (2002) 125-154.

[4] E. Alòs and S. Bonaccorsi, Stability for stochastic partial differential equations with Dirichlet white-noise boundary conditions. Infin. Dimens. Anal. Quantum Probab. Relat. Top. 5 (2002) 465-481.

[5] J.P. Aubin and H. Frankowska, Set-valued analysis, Systems \& Control: Foundations \& Applications 2. Birkhäuser Boston Inc., Boston, MA (1990).

[6] A.V. Balakrishnan, Applied functional analysis, Applications of Mathematics 3. Springer-Verlag, New York (1976).

[7] A. Chojnowska-Michalik, A semigroup approach to boundary problems for stochastic hyperbolic systems. Preprint (1978).

[8] G. Da Prato and J. Zabczyk, Evolution equations with white-noise boundary conditions. Stoch. Stoch. Rep. 42 (1993) $167-182$. 
[9] G. Da Prato and J. Zabczyk, Ergodicity for infinite-dimensional systems. London Math. Soc. Lect. Notes Ser. 229, Cambridge University Press (1996).

[10] T.E. Duncan, B. Maslowski and B. Pasik-Duncan, Ergodic boundary/point control of stochastic semilinear systems. SIAM J. Control Optim. 36 (1998) 1020-1047.

[11] N. El Karoui, S. Peng and M.C. Quenez, Backward stochastic differential equations in finance. Math. Finance 7 (1997) 1-71.

[12] H.O. Fattorini, Boundary control systems. SIAM J. Control 6 (1968) 349-385.

[13] W.H. Fleming and H.M. Soner, Controlled Markov processes and viscosity solutions. Appl. Math. 25, Springer-Verlag, New York (1993).

[14] M. Fuhrman and G. Tessitore, Nonlinear Kolmogorov equations in infinite dimensional spaces: the backward stochastic differential equations approach and applications to optimal control. Ann. Probab. 30 (2002) 1397-1465.

[15] M. Fuhrman and G. Tessitore, Infinite horizon backward stochastic differential equations and elliptic equations in Hilbert spaces. Ann. Probab. 32 (2004) 607-660.

[16] A.V. Fursikov and O.Y. Imanuvilov, Controllability of Evolution Equations. Lect. Notes Ser. 34, Seoul National University Research Institute of Mathematics Global Analysis Research Center, Seoul (1996).

[17] F. Gozzi, Regularity of solutions of second order Hamilton-Jacobi equations and application to a control problem. Comm. Part. Diff. Eq. 20 (1995) 775-826.

[18] F. Gozzi, Global regular solutions of second order Hamilton-Jacobi equations in Hilbert spaces with locally Lipschitz nonlinearities. J. Math. Anal. Appl. 198 (1996) 399-443.

[19] F. Gozzi, E. Rouy and A. Święch, Second order Hamilton-Jacobi equations in Hilbert spaces and stochastic boundary control. SIAM J. Control Optim. 38 (2000) 400-430.

[20] A. Grorud and E. Pardoux, Intégrales Hilbertiennes anticipantes par rapport à un processus de Wiener cylindrique et calcul stochastique associé. Appl. Math. Optim. 25 (1992) 31-49.

[21] A. Ichikawa, Stability of parabolic equations with boundary and pointwise noise, in Stochastic differential systems (MarseilleLuminy, 1984). Lect. Notes Control Inform. Sci. 69 (1985) 55-66.

[22] I. Lasiecka and R. Triggiani, Differential and algebraic Riccati equations with application to boundary/point control problems: continuous theory and approximation theory. Lect. Notes Control Inform. Sci. 164, Springer-Verlag, Berlin (1991).

[23] B. Maslowski, Stability of semilinear equations with boundary and pointwise noise. Ann. Scuola Norm. Sup. Pisa Cl. Sci. 22 (1995) 55-93.

[24] D. Nualart, The Malliavin Calculus and Related Topics, Probability and its Applications, Springer (1995).

[25] D. Nualart and E. Pardoux, Stochastic calculus with anticipative integrands. Probab. Th. Rel. Fields 78 (1988) 535-581.

[26] E. Pardoux and S. Peng, Adapted solution of a backward stochastic differential equation. Syst. Control Lett. 14 (1990) 55-61.

[27] E. Pardoux and S. Peng, Backward stochastic differential equations and quasilinear parabolic partial differential equations, in Stochastic partial differential equations and their applications, B.L. Rozowskii and R.B. Sowers Eds., Springer, Lect. Notes Control Inf. Sci. 176 (1992) 200-217.

[28] Y.A. Rozanov and Yu. A., General boundary value problems for stochastic partial differential equations. Trudy Mat. Inst. Steklov. 200 (1991) 289-298.

[29] R.B. Sowers, Multidimensional reaction-diffusion equations with white noise boundary perturbations. Ann. Probab. 22 (1994) (2071-2121)

[30] A. Święch, "Unbounded" second order partial differential equations in infinite-dimensional Hilbert spaces. Comm. Part. Diff. Eq. 19 (1994) 11-12, 1999-2036. 